\title{
Canonical formulation and path integral for local vacuum energy sequestering
}

\author{
R. Bufalo ${ }^{a *}$, J. Klusoñ ${ }^{b \dagger}$ and M. Oksanen ${ }^{c \ddagger}$ \\ ${ }^{a}$ Universidade Federal do ABC, Centro de Ciências Naturais e Humanas, \\ Av. dos Estados, 5001, 09210-580 Santo André, SP, Brazil \\ ${ }^{b}$ Department of Theoretical Physics and Astrophysics, Faculty of Science, \\ Masaryk University, Kotlářská 2, 611 37, Brno, Czech Republic \\ ${ }^{c}$ Department of Physics, University of Helsinki, P.O. Box 64 \\ FI-00014 Helsinki, Finland
}

\begin{abstract}
We establish the Hamiltonian analysis and the canonical path integral for a local formulation of vacuum energy sequestering. In particular, by considering the state of the universe as a superposition of vacuum states corresponding to different values of the cosmological and gravitational constants, the path integral is extended to include integrations over the cosmological and gravitational constants. The result is an extension of the Ng-van Dam form of the path integral of unimodular gravity. It is argued to imply a relation between the fraction of the most likely values of the gravitational and cosmological constants and the average values of the energy density and pressure of matter over spacetime. Finally, we construct and analyze a BRST-exact formulation of the theory, which can be considered as a topological field theory.
\end{abstract}

PACS: 04.20.Fy, 04.60.Gw, 04.50.Kd , 11.10.Ef.

\section{Introduction}

According to a convincing body of observations the expansion of the universe is accelerating. Thus, assuming that general relativity (GR) continues to describe the universe accurately at the largest scales, we observe that the cosmological constant is not zero. There are many proposals seeking to explain the smallness of the cosmological constant, although with no understanding of its fundamental origin. Unfortunately, the situation is not that simple as one might initially expect, because the theoretical and observational results fail to come to a unified answer, since the theoretical appraisal exceeds the observed value by 120 orders of magnitude. Furthermore, the cosmological

\footnotetext{
${ }^{*}$ E-mail: rodrigo.bufalo@ufabc.edu.br

${ }^{\dagger}$ E-mail: klu@physics.muni.cz

${ }_{\ddagger}^{\ddagger}$ E-mail: markku.oksanen@helsinki.fi
} 
constant predicted by quantum field theory (QFT) is radiatively unstable to the extreme. This is the so-called cosmological constant problem [1, 2], the worst but most important problem of fine-tuning in physics.

The origin of this outstanding disagreement can be traced back to the universality of gravity and the quantum generation of vacuum energy by virtual particles. In a quantum field theorist point-of-view even the vacuum possesses energy density, given by the resummation of the QFT bubble diagrams. In GR, vacuum energy contributes to the cosmological constant, and the vacuum geometry must be curved due to the equivalence principle.

On the one hand, if one approaches this problem by enforcing a symmetry principle, for instance supersymmetry and/or conformal symmetry, the huge vacuum energy could be canceled. However, at scales below a $\mathrm{TeV}$, these symmetries are broken. On the other hand, one could alternatively approach this situation by means of a dynamical adjustment of vacuum energy, where a nongravitating degree of freedom is responsible for "eating" it all up. The major issue with this idea is to work around Weinberg's no-go theorem [1] which prohibits such adjustment in any standard QFT coupled to gravity.

One of the first and best-known (minimal) modifications of GR that was hoped to shed new light on the cosmological constant problem is unimodular gravity [ 3 [ 6$]$. It is well known that the field equation for the metric in unimodular gravity is either the traceless Einstein equation or, thanks to the Bianchi identity, the Einstein equation with a cosmological constant [7]. Actually, the main conceptual difference to GR is that the cosmological constant of unimodular gravity is a constant of integration, rather than a coupling constant. This different point of view on the cosmological constant has led to considerable interest in several topics within the unimodular gravity scenario; see Refs. [8 13] and references therein. Unfortunately, a similar problem with the renormalization or fine-tuning of the cosmological constant is found as in GR [1, 2].

Among the several new proposals made in recent years, which attempt to resolve the cosmological constant problem via (minimal) modification of GR, we would like to call attention to a particular proposal called vacuum energy sequestering [14]. It includes a global mechanism for decoupling the vacuum energy generated by matter loops from gravity. Hence it appears to be able to evade Weinberg's no-go theorem.

In order to avoid the drawbacks of previous approaches - mainly the extreme sensitivity of the (diffeomorphism-allowed) contribution to the cosmological constant for any change of the matter sector parameters or addition of higher-order loop corrections, and the need for it to be tuned by hand order by order in perturbation theory to ensure a particular finite value of the cosmological constant - the vacuum energy sequestering theory provides by design that all quantum-generated vacuum energy contributions from a protected matter sector cancel completely from the gravitational equations of motion [14-16]. Thus, the only vacuum energy remaining that now sources gravity is a renormalized vacuum energy, which is automatically radiatively stable and fully consistent with the concept of renormalization in QFT. The sequestering mechanism works at each and every order in perturbation theory, so there is no need to retune the classical cosmological constant when higher-order loop corrections are included. Hence, one is left with a radiatively stable cosmological constant, which is completely independent of the vacuum energy contributions from the protected matter sector [15].

The core of this proposal may be thought of as understanding GR as a hybrid of unimodular gravity in conjunction with variational procedures [17] to fix values of global variables such as the cosmological constant. The main feature of the sequester- 
ing mechanism is engendered by means of a global term $\sigma\left(\frac{\lambda}{\eta^{4} \mu^{4}}\right)$, which is added to the Einstein-Hilbert action [see Eq. (2.1)], so that all scales in the matter sector are now functionals of the gauge-invariant four-volume element of the universe $\int d^{4} x \sqrt{-g}$ [14]. The prescription of the vacuum energy sequestering mechanism uses a global scaling symmetry, in terms of a scaling parameter measuring the matter sector scales in Planck units, as an organizing principle for accounting for all quantum vacuum energy contributions. This is the key point of the sequestering mechanism that provides the way around Weinberg's no-go theorem. Unlike in GR or in its unimodular formulation, now the four-volume $\int d^{4} x \sqrt{-g}$ is an independent variable. Remarkably, locally the sequestering theory behaves just like standard GR, in the (semi) classical limit, but without a large cosmological constant and without its radiative instability [15].

Although successfully explaining the net value and stability of the cosmological constant through a dynamical adjustment, the global term in the sequestering mechanism is unusual and appears to conflict with the expectations about the microscopic origin of the mechanism. In order to address this deficiency, a local formulation of the theory has been recently proposed [18, 19]. In this manifestly local version of the sequestering mechanism the global terms are regarded as conserved quantities, and gauge redundancies are introduced in order to allow for the rigid variables to become solutions of local field equations. Actually, this local setup might be obtained from the global one by using a similar reparametrization invariance approach as in the Henneaux-Teitelboim form of unimodular gravity [8].

Interestingly, by virtue of the local conservation laws, the modifications of the gravitational sector in the local sequestering mechanism behave very similarly to the global setup of [14]. However, now solutions display the new features of a finite, eternal cosmological constant, and the spacetime volume of the underlying geometry does not have to be finite, while supporting a finite Planck scale and (protected) matter sector scales. Recently, the local framework has been further explored to understand cosmological behavior, the effects of phase transitions, and the interplay between gravity and particle physics [19].

It has been speculated that the local setup of the sequestering mechanism admits a standard Hamiltonian dynamics, allowing thus a definition of the Feynman path integral. We therefore wish to establish a Hamiltonian formalism and determine the canonical path integral for the local version of vacuum energy sequestering. The path integral enables us to work out an interesting point regarding the gravitational and cosmological constants. The situation is similar to the case of unimodular gravity, where an additional integration over the cosmological constant can be included, meaning that we are integrating over different physical boundary conditions (vacua) [9, 10, 13]. We now extend the idea of the Ng-van Dam form of the path integral to encompass integration over both the cosmological and gravitational constants in the path integral of the local sequestering model.

We also explore the possibility of a topological or Becchi-Rouet-Stora-Tyutin (BRST)exact formulation of the local vacuum energy sequestering model. This approach is inspired by a recent work on a so-called topological induced gravity [20], which is shown to be a simple special case of the local formulation of vacuum energy sequestering in Sect. 5, and by a similar approach to unimodular gravity in [21] (see Sect. 7). We regard that the gravitational action of vacuum energy sequestering appears as a gauge-fixing action along with an appropriate ghost action. As a result the action of 
vacuum energy sequestering becomes BRST exact and can be considered as a topological field theory [22]. The canonical structure of the ghost sector and the BRST charges are fully analyzed.

The paper is organized as follows. In Sect. 2 we present the vacuum energy sequestering theory in the original (global) form and its local extension, and explain how the sequestering mechanism works. Section 3 is dedicated to the Hamiltonian formulation of the local theory. In Sect. 4 the canonical path integral is established and we show that it can written in an extended Ng-van Dam form. That enables us to obtain a relation between the cosmological and gravitational constants. In Sect. 5 we prove a relation between the topological induced gravity as a particular case of the local vacuum energy sequestering model. Section 6 conceives the local sequestering model in a BRST-exact form, and a canonical analysis of the resulting theory is performed. For the purpose of comparison, we discuss another attempt to cope with the cosmological constant problem [21] in Sect. 7, which includes the cosmological constant in terms of a topological field theory, but lacks a mechanism for ensuring its radiative stability. Final remarks are presented in Sect. 8.

\section{Vacuum energy sequestering}

\subsection{Global mechanism}

The vacuum energy sequestering mechanism is based on the presence of two rigid variables with no local degrees of freedom: the bare cosmological constant $\lambda$ and a scaling parameter $\eta$ measuring the matter sector scales in Planck units. The main result of this procedure is that it sets the boundary condition upon $\eta$ in such a way that at every order of the loop expansion it takes precisely the necessary value in order to completely cancel the vacuum energy contribution from the matter sector at that order. The action for the given theory can be expressed as [14]

$$
S=\int d^{4} x \sqrt{-g}\left(\frac{M_{\mathrm{P}}^{2}}{2} R-\lambda+\eta^{4} \mathcal{L}_{\mathrm{m}}\left(\eta^{-2} g_{\mu \nu}, \Psi\right)\right)+\sigma\left(\frac{\lambda}{\eta^{4} \mu^{4}}\right),
$$

where the global function $\sigma$ is required to be an odd differentiable function, and the mass scale $\mu$ is around the QFT cutoff. Matter fields - denoted collectively by $\Psi$ - are coupled minimally to the metric.

From the variation of Eq. (2.1) with respect to $\lambda$, which links the four-volume to the scaling parameter $\eta$, we find a necessary condition for the matter scales to be nonzero, representing the picture of a finite universe in spacetime, collapsing in the future [16]. In particular, if the matter sector is the Standard Model of elementary particles, the vacuum energy sequestering mechanism prevents it from generating large contributions to the net cosmological constant. The variation of Eq. (2.1) upon the metric $g_{\mu \nu}$ yields

$$
M_{\mathrm{P}}^{2} G_{\nu}^{\mu}=T_{\nu}^{\mu}-\frac{1}{4} \delta_{\nu}^{\mu}\left\langle T_{\alpha}^{\alpha}\right\rangle
$$

where $T_{\nu}^{\mu}$ is the energy-momentum tensor of the matter fields, and we have eliminated $\lambda$ by its constraint equation $\lambda=\frac{1}{4}\left\langle T_{\alpha}^{\alpha}\right\rangle$, where we have defined the four-volume average by $\langle P\rangle=\int d^{4} x \sqrt{-g} P / \int d^{4} x \sqrt{-g}$. Now we see that Eq. (2.2) is the key result of the sequestering mechanism. Furthermore, this field equation is unlike in unimodular gravity, where the restricted variation removes the trace equation that involves the 
vacuum energy, but returns it as an arbitrary integration constant. On the other hand, in the sequestering mechanism there are no hidden equations or integration constants, all the sources are automatically accounted for in the right-hand side of Eq. (2.2).

Let us now scrutinize the main feature from (2.2). Remarkably, the matter-sector quantum corrections to vacuum energy are all accounted for in the average $\left\langle T_{\alpha}^{\alpha}\right\rangle$, and cancel precisely from the right-hand side of Eq. (2.2). This can be better viewed by extracting the constant contribution into the stress energy, $V_{\mathrm{vac}}$, so that we can rewrite the stress energy tensor as $T_{\nu}^{\mu}=-V_{\text {vac }} \delta_{\nu}^{\mu}+\tau_{\nu}^{\mu}$, where the tensor $\tau_{\nu}^{\mu}$ describes local excitations. We therefore see that by means of such decomposition $V_{\text {vac }}$ completely drops out from (2.2) [14, 15]. Furthermore, this result shows that the only vacuum energy that sources gravity is a renormalized vacuum energy, which is automatically radiatively stable.

However, note that there is a residual cosmological constant left: the historic average $\left\langle\tau_{\alpha}^{\alpha}\right\rangle$ which is notably insensitive to vacuum loop corrections, and is precisely small in large and old universes by virtue of two approximate symmetries, the scalings $\eta \rightarrow \Omega \eta, g_{\mu \nu} \rightarrow \Omega^{-2} g_{\mu \nu}$, and $\lambda \rightarrow \Omega^{4} \lambda$, and the shifts $\lambda \rightarrow \lambda+\alpha \eta^{4}$ and $\mathcal{L}_{\mathrm{m}} \rightarrow \mathcal{L}_{\mathrm{m}}-\alpha$, which are broken only by the gravitational sector [15]. At last, as with any leftover of a UV-sensitive physical quantity in QFT, the numerical value of the finite part of the cosmological constant is not determined by the theory, but rather determined to match observations.

\subsection{Local formulation}

A local formulation of the sequestering mechanism has been proposed [18] mainly to deal with the microscopic origin of the mechanism, interpreting the global terms $\sigma\left(\frac{\lambda}{\eta^{4} \mu^{4}}\right)$ as conserved quantities, so that the rigid variables $\lambda, \eta$ are solutions of local field equations. As a first step on its definition, it is relevant for definiteness of the local formulation to absorb $\eta$ into the definition of the Planck scale in the action (2.1). This is achieved by the change of variables $g_{\mu \nu} \rightarrow \frac{\kappa^{2}}{M_{\mathrm{P}}^{2}} g_{\mu \nu}, \lambda \rightarrow\left(\frac{M_{\mathrm{P}}^{2}}{\kappa^{2}}\right)^{2} \lambda$ where a new variable $\kappa^{2}=\frac{M_{\mathrm{P}}^{2}}{\eta^{2}}$ is defined. In terms of this new variable, the action (2.2) now reads

$$
S=\int d^{4} x \sqrt{-g}\left(\frac{\kappa^{2}}{2} R-\lambda+\mathcal{L}_{\mathrm{m}}\left(g_{\mu \nu}, \Psi\right)\right)+\sigma\left(\frac{\lambda}{\mu^{4}}\right) .
$$

The variation of $\eta$ is now replaced by the $\kappa^{2}$ parameter, but the sequestering mechanism obtained from the field equations remains intact [18].

Now the path chosen to promote the rigid parameters $\kappa^{2}, \lambda$ to local fields, and reinterpret the global term as an integral of local expressions, which simultaneously yield local equations $\partial_{\mu} \kappa^{2}=0, \partial_{\mu} \lambda=0$ is very similar to the known gauge-invariant formulation of unimodular gravity by Henneaux and Teitelboim [8]. In their formulation, the unimodular constraint $\sqrt{-g}=\varepsilon_{0}$ is replaced by a diffeomorphism-invariant form $\sqrt{-g}=\partial_{\mu} \tau^{\mu}$ enforced by a term $-\int d^{4} \lambda(x)\left(\sqrt{-g}-\partial_{\mu} \tau^{\mu}\right)$, where $\lambda(x)$ is treated as a Lagrange multiplier satisfying $\partial_{\mu} \lambda=0$ and $\tau^{\mu}$ is a vector density. This diffeomorphisminvariant form can be derived from the global one via a parametrization of space-time coordinates [11] (see Refs. [17, 23] for a review).

Now, following a similar path for the local sequestering model, we wish to replace the $\lambda$-dependent terms in Eq. (2.3) with a (diffeomorphism-preserving) gauge-fixing 
action

$$
S_{g f}=-\int\left(\lambda \sqrt{-g} d^{4} x-d \hat{A} \hat{\sigma}\left(\frac{\lambda}{\mu^{4}}\right)\right)
$$

where $d \hat{A}$ is the exterior derivative of an auxiliary three-form $\hat{A}$ (see below). The new local additions should not gravitate directly in order to preserve the main feature of sequestering. On the one hand, the field equation obtained by varying $\hat{A}$ is precisely $\partial_{\mu} \lambda=0$, fixing the Lagrange multiplier $\lambda(x)$ to be an arbitrary rigid contribution to the total cosmological constant. On the other hand, the variation with respect to $\lambda(x)$ yields that $\hat{A}$ is a nonpropagating, auxiliary field. It should be clear that the real reason for the absence of any local degrees of freedom from $\lambda(x)$ is the newly introduced gauge redundancy of the three-form (see discussion below).

Since we have one more rigid Lagrange multiplier in Eq. (2.3),$\kappa^{2}$, we can follow exactly the same procedure to make it local off shell, and constant on shell by means of an extra copy of Eq. (2.4). So the action for local vacuum energy sequestering [18] can be written as (cf. Ref. [18])

$$
S=\int d^{4} x \sqrt{-g}\left(\frac{\kappa^{2}}{2} R-\lambda+\mathcal{L}_{\mathrm{m}}\left(g_{\mu \nu}, \Psi\right)\right)+\int d A \sigma\left(\frac{\kappa^{2}}{M_{\mathrm{P}}^{2}}\right)+\int d \hat{A} \hat{\sigma}\left(\frac{\lambda}{\mu^{4}}\right),
$$

where the bare gravitational and cosmological constants $\left(\kappa^{2}\right.$ and $\left.\lambda\right)$ are local fields. $d A$ and $d \hat{A}$ are exterior derivatives of two auxiliary three-forms $A$ and $\hat{A}$. Furthermore, $\sigma$ and $\hat{\sigma}$ are two smooth functions, where $M_{\mathrm{P}}$ and $\mu$ are the (cutoff) energy scales associated with gravity and matter, respectively. The measure four-forms can be written as

$$
\begin{aligned}
& d A=d^{4} x(* d A)=d^{4} x \frac{1}{4 !} \epsilon^{\mu \nu \rho \sigma} d A_{\mu \nu \rho \sigma}=d^{4} x \partial_{\mu} \omega^{\mu} \\
& d \hat{A}=d^{4} x(* d \hat{A})=d^{4} x \frac{1}{4 !} \epsilon^{\mu \nu \rho \sigma} d \hat{A}_{\mu \nu \rho \sigma}=d^{4} x \partial_{\mu} \tau^{\mu}
\end{aligned}
$$

where $\omega^{\mu}$ and $\tau^{\mu}$ are vector densities of unit weight:

$$
\omega^{\mu}=\frac{1}{3 !} \epsilon^{\mu \nu \rho \sigma} A_{\nu \rho \sigma}, \quad \tau^{\mu}=\frac{1}{3 !} \epsilon^{\mu \nu \rho \sigma} \hat{A}_{\nu \rho \sigma} .
$$

The four-forms are invariant under a gauge transformation $A \rightarrow A+d B$ and $\hat{A} \rightarrow \hat{A}+$ $d \hat{B}$, where $B$ and $\hat{B}$ are arbitrary two-forms. Equivalently, the gauge transformations of the measure can be written for the vector densities (2.8) as $\omega^{\mu} \rightarrow \omega^{\mu}+\partial_{\nu} \alpha^{\mu \nu}$ and $\tau^{\mu} \rightarrow \tau^{\mu}+\partial_{\nu} \hat{\alpha}^{\mu \nu}$, where $\alpha^{\mu \nu}$ and $\hat{\alpha}^{\mu \nu}$ are antisymmetric tensor densities of unit weight. In the presence of a metric we can further write $\omega^{\mu}=\sqrt{-g} U^{\mu}$ and $\tau^{\mu}=\sqrt{-g} V^{\mu}$, so that (similar to the situation in the fully diffeomorphism-invariant formulation of unimodular gravity [13])

$$
\partial_{\mu} \omega^{\mu}=\sqrt{-g} \nabla_{\mu} U^{\mu}, \quad \partial_{\mu} \tau^{\mu}=\sqrt{-g} \nabla_{\mu} V^{\mu}
$$

where $U^{\mu}$ and $V^{\mu}$ are vector fields. Hence we rewrite the action for local vacuum energy sequestering (2.5) as

$$
S=\int d^{4} x \sqrt{-g}\left[\frac{\kappa^{2}}{2} R-\lambda+\sigma\left(\frac{\kappa^{2}}{M_{\mathrm{P}}^{2}}\right) \nabla_{\mu} U^{\mu}+\hat{\sigma}\left(\frac{\lambda}{\mu^{4}}\right) \nabla_{\mu} V^{\mu}+\mathcal{L}_{\mathrm{m}}\left(g_{\mu \nu}, \Psi\right)\right] .
$$




\section{Canonical formulation of the local theory of vac- uum energy sequestering}

\subsection{ADM representation of the action}

The canonical formulation of the original vacuum energy sequestering model (2.1) [14] (see also Ref. [15]), where the sequestering of vacuum energy is achieved by including a (scaling) function outside of the action, has already been considered in Ref. [24]. For a canonical formulation of the local version of vacuum energy sequestering it is convenient to further rewrite the action (2.10) by partial integration as

$$
S=\int d^{4} x \sqrt{-g}\left[\frac{\kappa^{2}}{2} R-\lambda-\sigma^{\prime}\left(\frac{\kappa^{2}}{M_{\mathrm{P}}^{2}}\right) \frac{U^{\mu} \nabla_{\mu} \kappa^{2}}{M_{\mathrm{P}}^{2}}-\hat{\sigma}^{\prime}\left(\frac{\lambda}{\mu^{4}}\right) \frac{V^{\mu} \nabla_{\mu} \lambda}{\mu^{4}}+\mathcal{L}_{\mathrm{m}}\left(g_{\mu \nu}, \Psi\right)\right],
$$

where $\sigma^{\prime}$ and $\hat{\sigma}^{\prime}$ denote the first derivatives of the smooth functions $\sigma$ and $\hat{\sigma}$, which are assumed to be nonvanishing. In the simplest permitted case $\sigma^{\prime}$ and $\hat{\sigma}^{\prime}$ are constant (see Sec. 5). Since we are mostly interested in the propagating degrees of freedom and the role of the gravitational and cosmological constants, we omit all boundary terms. However, for the given form of the action (3.1) the boundary terms are of the same form as in GR (as well as in unimodular gravity [13]).

An Arnowitt-Deser-Misner (ADM) representation of the gravitational part of the action (3.1) is obtained as

$$
\begin{array}{r}
S_{\mathrm{ADM}}\left[N, N^{i}, h_{i j}, \kappa^{2}, U_{\boldsymbol{n}}, U^{i}, \lambda, V_{\boldsymbol{n}}, V^{i}\right]=\int d t d^{3} x N \sqrt{h}\left[\frac{\kappa^{2}}{2}\left(K_{i j} \mathcal{G}^{i j k l} K_{k l}+{ }^{(3)} R\right)\right. \\
-\left(K-\frac{\sigma^{\prime}}{M_{\mathrm{P}}^{2}} U_{\boldsymbol{n}}\right) \nabla_{n} \kappa^{2}-D_{i} D^{i} \kappa^{2}-\frac{\sigma^{\prime}}{M_{\mathrm{P}}^{2}} U^{i} \partial_{i} \kappa^{2} \\
\left.-\lambda+\frac{\hat{\sigma}^{\prime}}{\mu^{4}} V_{\boldsymbol{n}} \nabla_{n} \lambda-\frac{\hat{\sigma}^{\prime}}{\mu^{4}} V^{i} \partial_{i} \lambda\right]
\end{array}
$$

where the arguments of the functions $\sigma^{\prime}$ and $\hat{\sigma}^{\prime}$ are omitted, but their dependence on $\kappa^{2}$ and $\lambda$, respectively, should be kept in mind. The vector fields have been decomposed to components normal and tangent to the spatial hypersurface $\Sigma_{t}$, defined as

$$
U_{n}=n_{\mu} U^{\mu}, \quad U^{i}=\left(\delta_{\mu}^{i}+n^{i} n_{\mu}\right) U^{\mu}, \quad n^{\mu}=\left(\frac{1}{N}, \frac{N^{i}}{N}\right)
$$

where $n^{\mu}$ is the unit normal to $\Sigma_{t} \cdot 1$ The canonical formulation of the matter action is identical to that of GR.

\subsection{Hamiltonian and constraints}

Canonical momenta conjugate to $N, N^{i}, h_{i j}, \kappa^{2}, U_{\boldsymbol{n}}, U^{i}, \lambda, V_{\boldsymbol{n}}$ and $V^{i}$ are denoted by $\pi_{N}, \pi_{i}, \pi^{i j}, P_{\kappa^{2}}, P_{\boldsymbol{n}}, P_{i}, p_{\lambda}, p_{\boldsymbol{n}}$ and $p_{i}$, respectively. We obtain the primary constraints

$$
\pi_{N} \approx 0, \quad \pi_{i} \approx 0, \quad P_{\boldsymbol{n}} \approx 0, \quad P_{i} \approx 0, \quad p_{\boldsymbol{n}} \approx 0, \quad p_{i} \approx 0
$$

\footnotetext{
${ }^{1}$ Tensors and tensor densities that are tangent to the spatial hypersurfaces are denoted with latin indices $(i . j . .$.$) which run from 1$ to 3 . For a more detailed description of the notation see Ref. [13].
} 
and

$$
\mathcal{C}_{\lambda}=p_{\lambda}-\sqrt{h} \frac{\hat{\sigma}^{\prime}}{\mu^{4}} V_{\boldsymbol{n}} \approx 0
$$

The momenta conjugate to the metric $h_{i j}$ and $\kappa^{2}$ are defined as

$$
\pi^{i j}=\frac{1}{2} \sqrt{h} \kappa^{2} \mathcal{G}^{i j k l} K_{k l}-\frac{1}{2} \sqrt{h} h^{i j} \nabla_{n} \kappa^{2}
$$

and

$$
P_{\kappa^{2}}=-\sqrt{h}\left(K-\frac{\sigma^{\prime}}{M_{\mathrm{P}}^{2}} U_{n}\right) .
$$

The time derivatives of $\kappa^{2}$ and $h_{i j}$ are solved from Eqs. (3.6) and (3.7) as

$$
\nabla_{n} \kappa^{2}=\frac{1}{N}\left(\partial_{t} \kappa^{2}-N^{i} \partial_{i} \kappa^{2}\right)=-\frac{2}{3 \sqrt{h}}\left(\pi-\kappa^{2} P_{\kappa^{2}}+\sqrt{h} \frac{\kappa^{2}}{M_{\mathrm{P}}^{2}} \sigma^{\prime} U_{\boldsymbol{n}}\right)
$$

and

$$
K_{i j}=\frac{1}{2 N}\left(\partial_{t} h_{i j}-2 D_{(i} N_{j)}\right)=\frac{2 \mathcal{G}_{i j k l} \pi^{k l}}{\sqrt{h} \kappa^{2}}+\frac{h_{i j}}{3 \sqrt{h} \kappa^{2}}\left(\pi-\kappa^{2} P_{\kappa^{2}}+\sqrt{h} \frac{\kappa^{2}}{M_{\mathrm{P}}^{2}} \sigma^{\prime} U_{\boldsymbol{n}}\right)
$$

where $\pi=h_{i j} \pi^{i j}$.

The Hamiltonian is obtained as

$$
H=\int d^{3} x\left(N \mathcal{H}_{T}+N^{i} \mathcal{H}_{i}+u_{N} \pi_{N}+u_{N}^{i} \pi_{i}+u_{\lambda} \mathcal{C}_{\lambda}+u_{\boldsymbol{n}} P_{\boldsymbol{n}}+u^{i} P_{i}+v_{\boldsymbol{n}} p_{\boldsymbol{n}}+v^{i} p_{i}\right)
$$

where the so-called super-Hamiltonian and supermomentum are defined as

$$
\begin{aligned}
\mathcal{H}_{T}= & \frac{2 \pi^{i j} \mathcal{G}_{i j k l} \pi^{k l}}{\sqrt{h} \kappa^{2}}+\frac{1}{3 \sqrt{h} \kappa^{2}}\left(\pi-\kappa^{2} P_{\kappa^{2}}+\sqrt{h} \frac{\kappa^{2}}{M_{\mathrm{P}}^{2}} \sigma^{\prime} U_{n}\right)^{2}-\frac{1}{2} \sqrt{h} \kappa^{2(3)} R \\
& +\sqrt{h}\left(D_{i} D^{i} \kappa^{2}+\frac{\sigma^{\prime}}{M_{\mathrm{P}}^{2}} U^{i} \partial_{i} \kappa^{2}+\lambda+\frac{\hat{\sigma}^{\prime}}{\mu^{4}} V^{i} \partial_{i} \lambda\right)
\end{aligned}
$$

and

$$
\mathcal{H}_{i}=-2 h_{i j} D_{k} \pi^{j k}+\partial_{i} \kappa^{2} P_{\kappa^{2}}+\partial_{i} \lambda p_{\lambda}
$$

respectively, where we introduced the inverse De Witt metric as

$$
\mathcal{G}_{i j k l}=\frac{1}{2}\left(h_{i k} h_{j l}+h_{i l} h_{j k}\right)-\frac{1}{2} h_{i j} h_{k l},
$$

and $u_{N}, u_{N}^{i}, u_{\lambda}, u^{i}, u_{\boldsymbol{n}}, v^{i}, v_{\boldsymbol{n}}$ are unspecified Lagrange multipliers for the primary constraints.

Each primary constraint must be preserved under time evolution. For $\pi_{N} \approx 0$ and $\pi_{i} \approx 0$ we obtain the Hamiltonian constraint

$$
\mathcal{H}_{T} \approx 0
$$

and the momentum constraint

$$
\mathcal{H}_{i} \approx 0 \text {. }
$$

We extend the momentum constraint (3.12) with terms that are proportional to the primary constraints $P_{\boldsymbol{n}}$ and $p_{\boldsymbol{n}}$ so that the momentum constraint generates spatial 
diffeomorphisms for all the variables that are involved in the constraints 2 For that reason we redefine

$$
\mathcal{H}_{i}=-2 h_{i j} D_{k} \pi^{j k}+\partial_{i} \kappa^{2} P_{\kappa^{2}}+\partial_{i} \lambda p_{\lambda}+\partial_{i} U_{\boldsymbol{n}} P_{\boldsymbol{n}}+\partial_{i} V_{\boldsymbol{n}} p_{\boldsymbol{n}} \approx 0 .
$$

It is useful to define global (smeared) versions of the Hamiltonian and momentum constraints:

$$
\mathcal{H}_{T}[\xi]=\int d^{3} x \xi \mathcal{H}_{T}, \quad \Phi\left[\chi^{i}\right]=\int d^{3} x \chi^{i} \mathcal{H}_{i}
$$

The preservation of the constraints $P_{i} \approx 0$ and $p_{i} \approx 0$, is ensured by introducing the secondary constraints

$$
\begin{aligned}
\mathcal{B}_{i} & =\partial_{i} \kappa^{2} \approx 0, \\
\mathcal{C}_{i} & =\partial_{i} \lambda \approx 0 .
\end{aligned}
$$

These constraints imply that $\kappa^{2}$ and $\lambda$ are constant across space. We define smeared forms of $\mathcal{B}_{i}$ and $\mathcal{C}_{i}$ as

$$
\mathcal{B}\left[\chi^{i}\right]=\int d^{3} x \chi^{i} \partial_{i} \kappa^{2}, \quad \mathcal{C}\left[\chi^{i}\right]=\int d^{3} x \chi^{i} \partial_{i} \lambda .
$$

These constraints are included in the Hamiltonian (3.10) with Lagrange multipliers as $\mathcal{B}\left[u_{\kappa}^{i}\right]$ and $\mathcal{C}\left[v_{\lambda}^{i}\right]$; furthermore, the terms of $\mathcal{H}_{T}$ that are proportional to $\mathcal{B}_{i}$ and $\mathcal{C}_{i}$ are absorbed into the constraints $\mathcal{B}\left[u_{\kappa}^{i}\right]$ and $\mathcal{C}\left[v_{\lambda}^{i}\right]$ of the Hamiltonian. The preservation of the constraint $P_{\boldsymbol{n}} \approx 0$,

$$
\partial_{t} P_{\boldsymbol{n}}=\left\{P_{\boldsymbol{n}}, H\right\} \approx-\frac{2 N \sigma^{\prime}}{3 M_{\mathrm{P}}^{2}}\left(\pi-\kappa^{2} P_{\kappa^{2}}+\sqrt{h} \frac{\kappa^{2}}{M_{\mathrm{P}}^{2}} \sigma^{\prime} U_{\boldsymbol{n}}\right) \approx 0
$$

requires a new secondary constraint,

$$
\Pi=\pi-\kappa^{2} P_{\kappa^{2}}+\sqrt{h} \frac{\kappa^{2}}{M_{\mathrm{P}}^{2}} \sigma^{\prime} U_{\boldsymbol{n}} \approx 0 .
$$

The preservation of the constraint $p_{\boldsymbol{n}} \approx 0$,

$$
\partial_{t} p_{\boldsymbol{n}}=\left\{p_{\boldsymbol{n}}, H\right\} \approx \sqrt{h} \frac{\hat{\sigma}^{\prime}}{\mu^{4}} u_{\lambda} \approx 0
$$

is ensured by fixing the Lagrange multiplier $u_{\lambda}$ of the constraint $\mathcal{C}_{\lambda}$ as

$$
u_{\lambda}=0 .
$$

The preservation of the constraint (3.5),

$$
\partial_{t} \mathcal{C}_{\lambda}=\left\{\mathcal{C}_{\lambda}, H\right\} \approx\left\{\mathcal{C}_{\lambda}, \mathcal{H}_{T}[N]\right\}-\sqrt{h} \frac{\hat{\sigma}^{\prime}}{\mu^{4}} v_{\boldsymbol{n}}+\left\{\mathcal{C}_{\lambda}, \mathcal{C}\left[v_{\lambda}^{i}\right]\right\} \approx 0
$$

is ensured by fixing the Lagrange multiplier $v_{\boldsymbol{n}}$ of the constraint $p_{\boldsymbol{n}}$ as

$$
v_{\boldsymbol{n}}=-N \frac{\mu^{4}}{\hat{\sigma}^{\prime}}+\frac{N \pi V_{\boldsymbol{n}}}{\sqrt{h} \kappa^{2}}+\frac{\partial_{i} v_{\lambda}^{i}}{\sqrt{h}} \frac{\mu^{4}}{\hat{\sigma}^{\prime}} .
$$

\footnotetext{
${ }^{2}$ We do not include a generator for the variables $\left(U^{i}, P_{j}\right)$ and $\left(V^{i}, p_{j}\right)$ since the terms of the Hamiltonian constraints (3.11) that depend on $U^{i}$ and $V^{i}$ are proportional to the constraints (3.18) and (3.19), respectively.
} 
At this point the Hamiltonian is written as

$$
\begin{aligned}
H=\int d^{3} x\left(N \mathcal{H}_{T}^{\prime}+N^{i} \mathcal{H}_{i}+u_{N} \pi_{N}+u_{N}^{i} \pi_{i}+u_{\boldsymbol{n}} P_{\boldsymbol{n}}+u^{i} P_{i}\right. & +v^{i} p_{i}+u_{\Pi} \Pi \\
& \left.+u_{\kappa}^{i} \mathcal{B}_{i}+v_{\lambda}^{i} \mathcal{C}_{i}^{\prime}\right),
\end{aligned}
$$

where we have defined the constraints

$$
\begin{aligned}
\mathcal{H}_{T}^{\prime} & =\mathcal{H}_{T}-\frac{\mu^{4}}{\hat{\sigma}^{\prime}} p_{\boldsymbol{n}}+\frac{\pi V_{\boldsymbol{n}}}{\sqrt{h} \kappa^{2}} p_{\boldsymbol{n}} \approx 0, \\
\mathcal{H}_{T} & =\frac{2 \pi^{i j} \mathcal{G}_{i j k l} \pi^{k l}}{\sqrt{h} \kappa^{2}}-\frac{1}{2} \sqrt{h} \kappa^{2(3)} R+\sqrt{h} D_{i} D^{i} \kappa^{2}+\sqrt{h} \lambda \approx 0, \\
\mathcal{C}_{i}^{\prime} & =\mathcal{C}_{i}-\mu^{4} \partial_{i}\left(\frac{p_{\boldsymbol{n}}}{\sqrt{h} \hat{\sigma}^{\prime}}\right)=\partial_{i} \lambda-\mu^{4} \partial_{i}\left(\frac{p_{\boldsymbol{n}}}{\sqrt{h} \hat{\sigma}^{\prime}}\right) \approx 0
\end{aligned}
$$

and $u_{N}, u_{N}^{i}, u_{\boldsymbol{n}}, u^{i}, u_{\Pi}, u_{\kappa}^{i}, v^{i}, v_{\lambda}^{i}$ are unspecified Lagrange multipliers. Note that we have included the constraint (3.22) with a Lagrange multiplier as $\Pi\left[u_{\Pi}\right]=\int d^{3} x u_{\Pi} \Pi$ and absorbed the terms proportional to $\Pi$ from the Hamiltonian constraint. Rewriting the consistency condition for $P_{\boldsymbol{n}}$ with the Hamiltonian (3.27),

$$
\partial_{t} P_{\boldsymbol{n}} \approx\left\{P_{\boldsymbol{n}}, \Pi\left[u_{\Pi}\right]\right\}=-\sqrt{h} \frac{\kappa^{2}}{M_{\mathrm{P}}^{2}} \sigma^{\prime} u_{\Pi}=0,
$$

implies

$$
u_{\Pi}=0,
$$

i.e., the constraint $\Pi$ drops out of the Hamiltonian.

Then we must establish the preservation of the secondary constraints $\mathcal{H}_{T}, \mathcal{H}_{i}, \mathcal{B}_{i}$, $\mathcal{C}_{i}$ and $\Pi$. First we consider preservation of $\Pi$,

$$
\partial_{t} \Pi=\{\Pi, H\} \approx\left\{\Pi, \mathcal{H}_{T}[N]\right\}+\sqrt{h} \frac{\kappa^{2}}{M_{\mathrm{P}}^{2}} \sigma^{\prime} u_{\boldsymbol{n}}+\left\{\Pi, \mathcal{B}\left[u_{\kappa}^{i}\right]\right\} \approx 0,
$$

which is achieved by fixing the Lagrange multiplier $u_{\boldsymbol{n}}$ of the constraint $P_{\boldsymbol{n}}$ as

$$
u_{\boldsymbol{n}}=N \frac{M_{\mathrm{P}}^{2}}{\kappa^{2} \sigma^{\prime}}\left(\frac{3}{2} D_{i} D^{i} \kappa^{2}+2 \lambda\right)+N \frac{\pi U_{\boldsymbol{n}}}{\sqrt{h} \kappa^{2}}+\frac{3 M_{\mathrm{P}}^{2}}{2 \kappa^{2} \sigma^{\prime}} h^{i j} \partial_{i} N \partial_{j} \kappa^{2}+\frac{M_{\mathrm{P}}^{2}}{\sqrt{h} \sigma^{\prime}} \partial_{i} u_{\kappa}^{i} .
$$

The Hamiltonian is written as

$$
H=\int d^{3} x\left(N \mathcal{H}_{T}^{\prime \prime}+N^{i} \mathcal{H}_{i}+u_{N} \pi_{N}+u_{N}^{i} \pi_{i}+u^{i} P_{i}+v^{i} p_{i}+u_{\kappa}^{i} \mathcal{B}_{i}^{\prime}+v_{\lambda}^{i} \mathcal{C}_{i}^{\prime}\right)
$$

where we have defined a first-class Hamiltonian constraint as

$$
\begin{aligned}
\mathcal{H}_{T}^{\prime \prime}= & \mathcal{H}_{T}-\frac{\mu^{4}}{\hat{\sigma}^{\prime}} p_{\boldsymbol{n}}+\frac{\pi V_{\boldsymbol{n}}}{\sqrt{h} \kappa^{2}} p_{\boldsymbol{n}}+\frac{3 M_{\mathrm{P}}^{2}}{2\left(\kappa^{2} \sigma^{\prime}\right)^{2}} h^{i j} \partial_{i}\left(\kappa^{2} \sigma^{\prime}\right) \partial_{j} \kappa^{2} P_{\boldsymbol{n}} \\
& -\frac{3 M_{\mathrm{P}}^{2}}{2 \kappa^{2} \sigma^{\prime}} h^{i j} \partial_{i} \kappa^{2} D_{j} P_{\boldsymbol{n}}+\frac{2 M_{\mathrm{P}}^{2} \lambda}{\kappa^{2} \sigma^{\prime}} P_{\boldsymbol{n}}+\frac{\pi U_{\boldsymbol{n}}}{\sqrt{h} \kappa^{2}} P_{\boldsymbol{n}} \approx 0, \\
\mathcal{H}_{T}= & \frac{2 \pi^{i j} \mathcal{G}_{i j k l} \pi^{k l}}{\sqrt{h} \kappa^{2}}-\frac{1}{2} \sqrt{h} \kappa^{2(3)} R+\sqrt{h} D_{i} D^{i} \kappa^{2}+\sqrt{h} \lambda \approx 0 .
\end{aligned}
$$


and an extension of the constraint (3.18) as

$$
\mathcal{B}_{i}^{\prime}=\mathcal{B}_{i}-M_{\mathrm{P}}^{2} \partial_{i}\left(\frac{P_{\boldsymbol{n}}}{\sqrt{h} \sigma^{\prime}}\right)=\partial_{i} \kappa^{2}-M_{\mathrm{P}}^{2} \partial_{i}\left(\frac{P_{\boldsymbol{n}}}{\sqrt{h} \sigma^{\prime}}\right) \approx 0
$$

Proving the preservation of $\mathcal{H}_{T}, \mathcal{H}_{i}, \mathcal{B}_{i}$ and $\mathcal{C}_{i}$ is straightforward, since their structure is rather similar to that in Ref. [13]. Note that the Hamiltonian constraint does not depend on $P_{\kappa^{2}}$ and the constraints $\mathcal{H}_{T}, \mathcal{H}_{i}, \mathcal{B}_{i}, \mathcal{C}_{i}$ have vanishing Poisson brackets with both $P_{\boldsymbol{n}}$ and $p_{\boldsymbol{n}}$. The constraints $\mathcal{H}_{T}, \mathcal{H}_{i}, \mathcal{B}_{i}, \mathcal{C}_{i}$ satisfy the following Poisson brackets (the five omitted Poisson brackets vanish strongly):

$$
\begin{aligned}
\left\{\mathcal{H}_{T}[\xi], \mathcal{H}_{T}[\eta]\right\}= & \int d^{3} x\left(\xi \partial_{i} \eta-\eta \partial_{i} \xi\right) h^{i j}\left[\mathcal{H}_{j}-\left(P_{\kappa^{2}}+4 \kappa^{-2} \pi^{i j}-\kappa^{-2} h^{i j} \pi\right) \mathcal{B}_{j}\right. \\
& \left.-\partial_{j} U_{\boldsymbol{n}} p_{\boldsymbol{n}}-p_{\lambda} \mathcal{C}_{j}-\partial_{j} V_{\boldsymbol{n}} p_{\boldsymbol{n}}\right] \\
\left\{\Phi\left[\chi^{i}\right], \mathcal{H}_{T}[\xi]\right\}= & \mathcal{H}_{T}\left[\chi^{i} \partial_{i} \xi\right] \\
\left\{\Phi\left[\chi^{i}\right], \Phi\left[\psi^{j}\right]\right\}= & \Phi\left[\chi^{j} \partial_{j} \psi^{i}-\psi^{j} \partial_{j} \chi^{i}\right] \\
\left\{\Phi\left[\chi^{i}\right], \mathcal{B}\left[\eta^{j}\right]\right\}= & \mathcal{B}\left[\chi^{i} \partial_{j} \eta^{j}\right] \\
\left\{\Phi\left[\chi^{i}\right], \mathcal{C}\left[\eta^{j}\right]\right\}= & \mathcal{C}\left[\chi^{i} \partial_{j} \eta^{j}\right] .
\end{aligned}
$$

Hence all the constraints are now consistent under time evolution.

The total Hamiltonian (3.35) is a sum of the first class constraints $\mathcal{H}_{T}^{\prime \prime}, \mathcal{H}_{i}, \pi_{N}, \pi_{i}$, $P_{i}, p_{i}, \mathcal{B}_{i}^{\prime}$ and $\mathcal{C}_{i}^{\prime}$. In addition, we have four second class constraints $p_{\boldsymbol{n}}, \mathcal{C}_{\lambda}, P_{\boldsymbol{n}}$ and $\Pi$.

\subsection{Counting of physical degrees of freedom}

In order to clarify the nature of the constraint (3.18) on $\kappa^{2}$, we decompose the variables $\kappa^{2}, P_{\kappa^{2}}$ as

$$
\begin{aligned}
\kappa^{2}(t, x) & =\kappa_{0}^{2}(t)+\overline{\kappa^{2}}(t, x), \\
P_{\kappa^{2}}(t, x) & =\frac{\sqrt{h}}{\int d^{3} x \sqrt{h}} P_{\kappa^{2}}^{0}(t)+\overline{P_{\kappa^{2}}}(t, x),
\end{aligned}
$$

where the zero modes describe the time-dependent average values of $\kappa^{2}$ and $P_{\kappa^{2}}$ over space,

$$
\kappa_{0}^{2}(t)=\frac{1}{\int d^{3} x \sqrt{h}} \int d^{3} x \sqrt{h} \kappa^{2}(t, x), \quad P_{\kappa^{2}}^{0}(t)=\int d^{3} x P_{\kappa^{2}}(t, x),
$$

and the oscillating modes have vanishing average values over space,

$$
\int d^{3} x \sqrt{h \kappa^{2}}(t, x)=0, \quad \int d^{3} x \overline{P_{\kappa^{2}}}(t, x)=0 .
$$

Such a decomposition can always be performed, but for infinite spaces care must be taken in imposing appropriate (asymptotic) boundary conditions and in defining the integrals over infinite volumes (see Refs.[13, 11]). The zero modes satisfy the canonical Poisson bracket

$$
\left\{\kappa_{0}^{2}, P_{\kappa^{2}}^{0}\right\}=1,
$$

while the oscillating modes satisfy

$$
\left\{\overline{\kappa^{2}}(x), \overline{P_{\kappa^{2}}}(y)\right\}=\delta(x-y)-\frac{\sqrt{h}(y)}{\int d^{3} z \sqrt{h}} \equiv \overline{\delta(x, y)},
$$


where we have defined the overlined $\delta$ function that satisfies 3

$$
\int d^{3} x \overline{\delta(x, y)} \sqrt{h}(x) f(x)=\sqrt{h}(y) \overline{f(y)}, \quad \int d^{3} y \overline{\delta(x, y)} f(y)=\overline{f(x)} .
$$

The Poisson brackets between zero modes and oscillating modes vanish

$$
\left\{\kappa_{0}^{2}, \overline{P_{\kappa^{2}}}(x)\right\}=0, \quad\left\{\overline{\kappa^{2}}(x), P_{\kappa^{2}}^{0}\right\}=0 .
$$

The cosmological constant variables $\left(\lambda, p_{\lambda}\right)$ are decomposed in the same way to zero modes $\left(\lambda_{0}, p_{\lambda}^{0}\right)$ and oscillating modes $\left(\bar{\lambda}, \overline{p_{\lambda}}\right)$. The purpose of the decomposition of the variables $\lambda$ and $\kappa^{2}$ is to separate the spatially oscillating components which vanish due to the constraints (3.18) and (3.19). When the variables $\left(\kappa^{2}, P_{\kappa^{2}}\right)$ and $\left(\lambda, p_{\lambda}\right)$ are decomposed, the constraints (3.18) and (3.19) can indeed be replaced with local constraints

$$
\overline{\mathcal{B}}=\overline{\kappa^{2}} \approx 0, \quad \overline{\mathcal{C}}=\bar{\lambda} \approx 0,
$$

since $\partial_{i} \kappa^{2}=\partial_{i} \overline{\kappa^{2}}=0$ implies that $\overline{\kappa^{2}}$ is constant over space and the zero-average condition (3.42) requires the constant to be zero. The corresponding first class constraints (3.30) and (3.38) are replaced with

$$
\begin{aligned}
& \overline{\mathcal{B}}^{\prime}=\overline{\kappa^{2}}-M_{\mathrm{P}}^{2} \overline{\left(\frac{P_{\boldsymbol{n}}}{\sqrt{h} \sigma^{\prime}}\right)} \approx 0, \\
& \overline{\mathcal{C}}^{\prime}=\bar{\lambda}-\mu^{4} \overline{\left(\frac{p_{\boldsymbol{n}}}{\sqrt{h} \hat{\sigma}^{\prime}}\right)} \approx 0,
\end{aligned}
$$

where the overline denotes a component whose integral over space vanishes.

The number of physical degrees of freedom is readily obtained via Dirac's formula. There are two propagating physical degrees of freedom for the graviton and two zero modes. The zero modes are the gravitational and cosmological constants, which do not evolve in time since the Hamiltonian does not depend on the corresponding canonical momenta.

\subsection{Elimination of auxiliary variables and classical equiva- lence to GR}

Gauge-fixing conditions associated with the generators (3.47) and (3.48) can be chosen as

$$
\overline{P_{\kappa^{2}}} \approx 0, \quad \overline{p_{\lambda}} \approx 0 .
$$

Furthermore we choose the gauge conditions for the generators $P_{i} \approx 0$ and $p_{i} \approx 0$ as

$$
U^{i} \approx 0, \quad V^{i} \approx 0
$$

Then we have a set of second class constraints as

$$
\phi_{I}=\left[p_{\boldsymbol{n}}, \mathcal{C}_{\lambda}, P_{\boldsymbol{n}}, \Pi, \overline{\mathcal{B}}^{\prime}, \overline{P_{\kappa^{2}}}, \overline{\mathcal{C}}^{\prime}, \overline{p_{\lambda}}, P_{i}, U^{i}, p_{i}, V^{i}\right]
$$

where $I=1, \ldots, 12$. We shall use these constraints for the elimination of the variables $V_{\boldsymbol{n}}, p_{\boldsymbol{n}}, U_{\boldsymbol{n}}, P_{\boldsymbol{n}}, \overline{\kappa^{2}}, \overline{P_{\kappa^{2}}}, \bar{\lambda}, \overline{p_{\lambda}}, U^{i}, P_{i}, V^{i}$ and $p_{i}$. In order to set the second class

\footnotetext{
${ }^{3}$ Note that $\overline{\delta(x, y)} \neq \overline{\delta(y, x)}$.
} 
constraints to zero strongly, we replace the Poisson bracket with the Dirac bracket. The matrix of Poisson brackets for the constraints (3.51),

$$
C_{I J}(x, y)=\left\{\phi_{I}(x), \phi_{J}(y)\right\}
$$

has the following nonvanishing components with $I<J$ [Poisson brackets that turn out to be proportional to the constraints (3.51) - and thus vanish - are omitted as well]

$$
\begin{aligned}
& C_{12}(x, y)=\left\{p_{\boldsymbol{n}}(x), \mathcal{C}_{\lambda}(y)\right\}=\sqrt{h} \frac{\hat{\sigma}^{\prime}}{\mu^{4}}(y) \delta(x-y), \\
& C_{24}(x, y)=\left\{\mathcal{C}_{\lambda}(x), \Pi(y)\right\}=-\frac{3}{2} \sqrt{h} \frac{\hat{\sigma}^{\prime}}{\mu^{4}} V_{\boldsymbol{n}}(x) \delta(x-y), \\
& C_{28}(x, y)=\left\{\mathcal{C}_{\lambda}(x), \overline{p_{\lambda}}(y)\right\}=-\sqrt{h} \frac{\hat{\sigma}^{\prime \prime}}{\mu^{8}} V_{\boldsymbol{n}}(x) \overline{\delta(x, y)} \\
& C_{34}(x, y)=\left\{P_{\boldsymbol{n}}(x), \Pi(y)\right\}=-\sqrt{h} \frac{\kappa^{2}}{M_{\mathrm{P}}^{2}} \sigma^{\prime}(y) \delta(x-y), \\
& C_{46}(x, y)=\left\{\Pi(x), \overline{P_{\kappa^{2}}}(y)\right\}=\frac{3}{2} \frac{\sqrt{h}(y) \overline{\delta(y, x)}}{\int d^{3} z \sqrt{h}} P_{\kappa^{2}}^{0}-\overline{\delta(x, y)} P_{\kappa^{2}}(x) \\
& C_{48}(x, y)=\left\{\Pi(x), \overline{p_{\lambda}}(y)\right\}=\frac{3}{2} \frac{\sqrt{h}\left(\frac{\sigma^{\prime}}{M_{\mathrm{P}}^{2}}+\frac{\kappa^{2} \sigma^{\prime \prime}}{M_{\mathrm{P}}^{4}}\right) \overline{(x) U_{\boldsymbol{n}}(x) \overline{\delta(x, x)}} p_{\lambda}^{0},}{d^{3} z \sqrt{h}}, \\
& C_{56}(x, y)=\left\{\overline{\mathcal{B}}^{\prime}(x), \overline{P_{\kappa^{2}}}(y)\right\}=\overline{\delta(x, y)}+\left(P_{\boldsymbol{n}^{-}} \text {-terms)} \approx \overline{\delta(x, y)},\right. \\
& C_{78}(x, y)=\left\{\overline{\mathcal{C}}^{\prime}(x), \overline{p_{\lambda}}(y)\right\}=\overline{\delta(x, y)}+\left(p_{\boldsymbol{n}^{-}} \text {terms }\right) \approx \overline{\delta(x, y)} \\
& C_{9,10}(x, y)=-\delta(x-y), \\
& C_{11,12}(x, y)=-\delta(x-y),
\end{aligned}
$$

where $\sigma^{\prime \prime}$ and $\hat{\sigma}^{\prime \prime}$ are the second derivatives the scale functions $\sigma$ and $\hat{\sigma}$. In the components of Eq. (3.52) with $I>J$ the coordinates $(x, y)$ are interchanged, e.g.

$$
C_{82}(x, y)=\left\{\overline{p_{\lambda}}(x), \mathcal{C}_{\lambda}(y)\right\}=\sqrt{h} \frac{\hat{\sigma}^{\prime \prime}}{\mu^{8}} V_{\boldsymbol{n}}(y) \overline{\delta(y, x)} .
$$

Also notice that when the constraints (3.51) are imposed strongly, the arguments of the scale functions and their derivatives now involve only the zero modes of $\kappa^{2}$ and $\lambda$ :

$$
\sigma\left(\frac{\kappa^{2}}{M_{\mathrm{P}}^{2}}\right)=\sigma\left(\frac{\kappa_{0}^{2}}{M_{\mathrm{P}}^{2}}\right), \quad \hat{\sigma}\left(\frac{\lambda}{\mu^{4}}\right)=\hat{\sigma}\left(\frac{\lambda_{0}}{\mu^{4}}\right), \quad \text { etc. }
$$

The inverse matrix $C_{I J}^{-1}(x, y)$ is defined by

$$
\begin{aligned}
& \sum_{J=1}^{12} \int d^{3} y C_{I J}^{-1}(x, y) C_{J K}(y, z)=\delta_{I K} \delta(x-z), \\
& \sum_{J=1}^{12} \int d^{3} y C_{I J}(x, y) C_{J K}^{-1}(y, z)=\delta_{I K} \delta(x-z) .
\end{aligned}
$$


The Dirac bracket is defined as

$$
\left\{f_{1}, f_{2}\right\}_{\mathrm{D}}=\left\{f_{1}, f_{2}\right\}-\sum_{I, J=1}^{12} \int d^{3} x d^{3} y\left\{f_{1}, \phi_{I}(x)\right\} C_{I J}^{-1}(x, y)\left\{\phi_{J}(y), f_{2}\right\},
$$

where $f_{1}$ and $f_{2}$ are any functions or functionals of the canonical variables. Since the nonvanishing components of the matrix $C_{I J}^{-1}(x, y)$ are the components with the indices $\{I J\}=\{12\},\{13\},\{17\},\{34\},\{35\},\{37\},\{56\},\{78\},\{9,10\},\{11,12\}$ and the corresponding components with $I<J$, we see that the Dirac bracket is equal to the Poisson bracket,

$$
\left\{f_{1}, f_{2}\right\}_{\mathrm{D}}=\left\{f_{1}, f_{2}\right\}
$$

for any $f_{1}$ and $f_{2}$ that depend on the remaining canonical variables $N, N^{i}, h_{i j}, \kappa_{0}^{2}, \lambda_{0}$, $\pi_{N}, \pi_{i}, \pi^{i j}, P_{\kappa^{2}}^{0}$ and $p_{\lambda}^{0}$. The Hamiltonian reduces to the GR form

$$
H=\int d^{3} x\left(N \mathcal{H}_{T}+N^{i} \mathcal{H}_{i}+u_{N} \pi_{N}+u_{N}^{i} \pi_{i}\right)
$$

where

$$
\begin{aligned}
\mathcal{H}_{T} & =\frac{2 \pi^{i j} \mathcal{G}_{i j k l} \pi^{k l}}{\kappa_{0}^{2} \sqrt{h}}-\frac{\kappa_{0}^{2}}{2} \sqrt{h^{(3)}} R+\sqrt{h} \lambda_{0}, \\
\mathcal{H}_{i} & =-2 h_{i j} D_{k} \pi^{j k}
\end{aligned}
$$

The gravitational and cosmological constants $\kappa_{0}^{2}$ and $\lambda_{0}$ depend on time formally, but they do not evolve since $\mathcal{H}_{T}$ is independent of $P_{\kappa^{2}}^{0}$ and $p_{\lambda}^{0}$.

\subsection{Gauge-fixed action for quantization}

Next we construct the path integral and the gauge-fixed action for the BRST formalism. Since the constraints $(\underline{3.30})$ and $(3.38)$ are total derivatives and their integrals vanish, we have linearly dependent generators. The quantization of a gauge system with linearly dependent generators is achieved in the formalism of Ref. [25]. Fortunately, the situation with the nonlocally linearly dependent generator associated with the (cosmological constant) variable $\lambda$ is similar to the case of unimodular gravity, which has been described in Ref. [13]. The generator associated with the (bare gravitational constant) variable $\kappa^{2}$ can be treated in a similar way in the formalism of Ref. [25].

First we solve the second-class constraints $\left(p_{\boldsymbol{n}}, \mathcal{C}_{\lambda}, P_{\boldsymbol{n}}, \Pi\right)$ and eliminate the variables $V_{\boldsymbol{n}}, p_{\boldsymbol{n}}, U_{\boldsymbol{n}}$ and $P_{\boldsymbol{n}}$. As shown in the previous subsection, the Dirac bracket is equal to the Poisson bracket for the remaining variables. The generators are denoted by

$$
G_{\alpha}=\left[\pi_{N}, \pi_{i}, \mathcal{H}_{T}, \mathcal{H}_{i}, \overline{\mathcal{B}}, \overline{\mathcal{C}}, P_{i}, p_{i}\right]
$$

and their nonvanishing Poisson/Dirac brackets are given in Eq. (3.39). Here the Hamiltonian and momentum constraints are defined in Eqs. (3.12) and (3.29). The gauge conditions are written as

$$
\chi^{\alpha}=\left[\sigma_{N}^{0}, \sigma_{N}^{i}, \chi^{0}, \chi^{i}, \overline{P_{\kappa^{2}}}, \overline{p_{\lambda}}, U^{i}, V^{i}\right],
$$

where $\sigma_{N}^{\mu}$ fix the lapse and shift functions, $\chi^{\mu}$ are coordinate conditions for the metric, and the conditions $\overline{P_{\kappa^{2}}}, \overline{p_{\lambda}}, U^{i}$ and $V^{i}$ are chosen for simplicity. Note that the gauge 
conditions for the generators (3.46) have to be degenerate to the same degree as the generators; in this case, the integrals of the conditions over space are fixed.

When the generators $G_{\alpha}$ are linearly dependent, there exist right zero eigenvectors $Z_{a}^{\alpha}$

$$
G_{\alpha} Z_{a}^{\alpha}=0
$$

The condensed index $\alpha$ labels each local generator at every point on the spatial hypersurfaces. Summing over such an index involves an integration over space in addition to a sum over the components. The latin index $a$ labels the zero eigenvectors. The vectors $Z_{a}^{\alpha}$ are assumed to be linearly independent, i.e., we consider a first-stage reducible theory. The gauge conditions $\chi^{\alpha}$ have to be similarly dependent as the generators, so that there exist left zero eigenvectors $\hat{Z}_{\alpha}^{a}$,

$$
\hat{Z}_{\alpha}^{a} \chi^{\alpha}=0
$$

The eigenvectors $Z_{a}^{\alpha}$ and $\hat{Z}_{\alpha}^{a}$ are the right and left zero vectors of the degenerate Faddeev-Popov operator,

$$
Q_{\beta}^{\alpha}=\left\{\chi^{\alpha}, G_{\beta}\right\},
$$

respectively. In this case, we have the two right eigenvectors 4

$$
\begin{aligned}
& Z_{1}^{\alpha}=\left[0,0,0,0, \frac{\sqrt{h}}{\int d^{3} x \sqrt{h}}, 0,0,0\right], \\
& Z_{2}^{\alpha}=\left[0,0,0,0,0, \frac{\sqrt{h}}{\int d^{3} x \sqrt{h}}, 0,0\right],
\end{aligned}
$$

and the left eigenvectors

$$
\begin{aligned}
\hat{Z}_{\alpha}^{1} & =[0,0,0,0,1,0,0,0], \\
\hat{Z}_{\alpha}^{2} & =[0,0,0,0,0,1,0,0] .
\end{aligned}
$$

Hence the Faddeev-Popov ghosts $c^{\alpha}, b_{\alpha}$ become gauge fields that require additional gauge fixing. For that purpose the set of ghosts and Lagrange multipliers $\left(c^{\alpha}, b_{\alpha}, \eta_{\alpha}\right)$ is extended to

$$
\Phi_{\mathrm{g}}=\left(c^{\alpha}, b_{\alpha}, \eta_{\alpha}, C^{a}, B_{a}, E^{a}, \theta_{a}, \vartheta^{a}\right),
$$

where $c^{\alpha}, b_{\alpha}, \theta_{a}, \vartheta^{a}$ are Grassmann anticommuting variables and the rest are commuting variables. The path integral and the corresponding effective gauge-fixed action are written as

$$
\begin{aligned}
Z= & \int \mathcal{D} q^{A} \mathcal{D} p_{A} \mathcal{D} \Phi_{\mathrm{g}} \exp \left[i\left(S+S_{\mathrm{gh}+\mathrm{gf}}\right)\right] \\
S_{\mathrm{gh}+\mathrm{gf}}= & -\int d t\left[b_{\alpha} Q^{\alpha}{ }_{\beta} c^{\beta}+B_{a}\left(\omega_{\alpha}^{a} Z_{b}^{\alpha}\right) C^{b}+\eta_{\alpha}\left(\chi^{\alpha}+\sigma_{a}^{\alpha} E^{a}\right)\right. \\
& \left.+\theta_{a} \omega_{\alpha}^{a} c^{\alpha}+b_{\alpha} \sigma_{a}^{\alpha} \vartheta^{a}\right]
\end{aligned}
$$

where $q^{A}$ and $p_{A}$ denote all the gauge fields and their canonically conjugated momenta, and $S$ is the action without gauge fixing. The extra Lagrange multipliers $\left(\theta_{a}, \vartheta^{a}\right)$

\footnotetext{
${ }^{4}$ The components of these eigenvectors match those in (3.62), so that for the generators with an index, $i=1,2,3$, the corresponding component of the eigenvector is understood to be repeated three times.
} 
impose the gauge conditions $\omega_{\alpha}^{a} c^{\alpha}=0$ and $b_{\alpha} \sigma_{a}^{\alpha}=0$ on the Faddeev-Popov ghosts, where the gauge parameters $\left(\omega_{\alpha}^{a}, \sigma_{a}^{\alpha}\right)$ are arbitrary. The variables $B_{a}$ and $C^{a}$ are the ghosts for the Faddeev-Popov ghost fields. The so-called extra ghosts $E^{a}$ regulate divergent factors $\delta(0)$ that appear in the original gauge fixing $\delta\left(\chi^{\alpha}\right)$ with a redundant set of gauge conditions (3.63). In our case, we can choose the gauge-fixing parameters for the ghosts as

$$
\begin{aligned}
\omega_{\alpha}^{1} & =[0,0,0,0,-1,0,0,0], \\
\omega_{\alpha}^{2} & =[0,0,0,0,0,-1,0,0], \\
\sigma_{1}^{\alpha} & =\left[0,0,0,0, \frac{\sqrt{h}}{\int d^{3} x \sqrt{h}}, 0,0,0\right], \\
\sigma_{2}^{\alpha} & =\left[0,0,0,0,0, \frac{\sqrt{h}}{\int d^{3} x \sqrt{h}}, 0,0\right],
\end{aligned}
$$

Integration over the ghost sector gives the path integral as

$$
Z=\int \mathcal{D} q^{A} \mathcal{D} p_{A} \frac{\operatorname{det} \mathcal{F}_{\beta}^{\alpha}}{\operatorname{det} q_{b}^{a} \operatorname{det} \hat{q}_{b}^{a}} \int \mathcal{D} E^{a} \delta\left(\chi^{\alpha}+\sigma_{a}^{\alpha} E^{a}\right)\left(\operatorname{det} \hat{q}_{b}^{a}\right) \exp (i S),
$$

where the gauge-fixed Faddeev-Popov operator is defined as

$$
\mathcal{F}_{\beta}^{\alpha}=Q_{\beta}^{\alpha}+\sigma_{a}^{\alpha} \omega_{\beta}^{a},
$$

and the following matrices are introduced

$$
q_{b}^{a}=\omega_{\alpha}^{a} Z_{b}^{\alpha}, \quad \hat{q}_{b}^{a}=\hat{Z}_{\alpha}^{a} \sigma_{b}^{\alpha} .
$$

The path integral (3.72) is independent of the chosen gauge parameters $\left(\omega_{\alpha}^{a}, \sigma_{a}^{\alpha}\right)$, since both the ratio of determinants $\left(\operatorname{det} \mathcal{F}_{\beta}^{\alpha} / \operatorname{det} q_{b}^{a} \operatorname{det} \hat{q}_{b}^{a}\right)$ and the regulated gauge-fixing factor are invariant under a change of the gauge parameters.

Since in the present case we chose the gauge fixing of the ghosts so that

$$
q_{b}^{a}=\left(\begin{array}{cc}
-1 & 0 \\
0 & -1
\end{array}\right), \quad \hat{q}_{b}^{a}=\left(\begin{array}{ll}
1 & 0 \\
0 & 1
\end{array}\right)
$$

the gauge fixing and ghost action for the path integral can be written in a simpler form without the additional ghosts and Lagrange multipliers as

$$
S_{\mathrm{gh}+\mathrm{gf}}=-\int d t\left[b_{\alpha} \mathcal{F}_{\beta}^{\alpha} c^{\beta}+\eta_{\alpha}\left(\chi^{\alpha}+\sigma_{a}^{\alpha} E^{a}\right)\right] .
$$

Furthermore we can trivially integrate over the parts of the ghost sector that correspond to the generators $\pi_{N}, \pi_{i}, P_{i}$ and $p_{i}$, absorbing them into the normalization of the path integral, so that the the gauge fixing and ghost action is written as

$$
\begin{aligned}
S_{\mathrm{gh}+\mathrm{gf}}=-\int d t\left(b_{\mu} Q_{\nu}^{\mu} c^{\nu}+\bar{b}_{m} \bar{Q}_{\mu}^{m} c^{\mu}+\bar{b}_{m} \overline{\mathcal{F}}_{n}^{m} \bar{c}^{n}+\eta_{\mu} \chi^{\mu}+\bar{\eta}_{m} \bar{\chi}^{m}\right. & \\
& \left.+\frac{\int d^{3} x \sqrt{h} \bar{\eta}_{m}}{\int d^{3} x \sqrt{h}} E^{m}\right)
\end{aligned}
$$


where we denote

$$
\begin{aligned}
\bar{\chi}^{m} & =\left[\overline{P_{\kappa^{2}}}, \overline{p_{\lambda}}\right], \\
\bar{G}_{m} & =[\overline{\mathcal{B}}, \overline{\mathcal{C}}],
\end{aligned}
$$

and we have defined

$$
\begin{aligned}
Q^{\mu}{ }_{\nu} & =\left\{\chi^{\mu}, \mathcal{H}_{\nu}\right\}, \quad \mathcal{H}_{\nu}=\left(\mathcal{H}_{T}, \mathcal{H}_{i}\right), \\
\bar{Q}^{m}{ }_{\mu} & =\left\{\bar{\chi}^{m}, \mathcal{H}_{\mu}\right\}, \\
\overline{\mathcal{F}}_{n}^{m} & =\left\{\bar{\chi}^{m}(x), \bar{G}_{n}(y)\right\}-\delta_{n}^{m} \frac{\sqrt{h}(x)}{\int d^{3} z \sqrt{h}}=-\delta^{m}{ }_{n} \delta(x-y) .
\end{aligned}
$$

Summing over the repeated indices in Eq. (3.77), $\mu, \nu=0,1,2,3$ and $m, n=1,2$, includes integration over space. The ghosts $b_{\mu}, c^{\mu}$ are associated with diffeomorphisms and the ghosts $\bar{b}_{m}, \bar{c}^{m}$ with the generators $\overline{\mathcal{B}}$ and $\overline{\mathcal{C}}$. Integration over the extra ghost $E^{m}$ imposes $\bar{\eta}_{m}$ to have a vanishing zero mode. We obtain the operator (3.81) for the gauge conditions (3.78) as

$$
\begin{aligned}
\bar{Q}_{0}^{1}(x, y)= & \overline{\delta(y, x)}\left(\frac{2 \pi^{i j} \mathcal{G}_{i j k l} \pi^{k l}}{\sqrt{h}\left(\kappa^{2}\right)^{2}}+\frac{1}{2} \sqrt{h^{(3)} R}\right)(y) \\
& -\overline{\delta(y, x)} \frac{\partial}{\partial y^{i}}\left(\sqrt{h}(y) h^{i j}(y) \frac{\partial}{\partial y^{j}}\right) \\
& +\overline{\delta(y, x)} \frac{\pi(y) P_{\kappa^{2}}^{0}}{\kappa^{2}(y) \int d^{3} z \sqrt{h}}, \\
\bar{Q}_{i}^{1}(x, y)= & \overline{\delta(y, x)}\left(\partial_{i} P_{\kappa^{2}}(y)+P_{\kappa^{2}}(y) \frac{\partial}{\partial y^{i}}\right) \\
& -\overline{\delta(y, x)} \frac{P_{\kappa^{2}}^{0}}{\int d^{3} z \sqrt{h}}\left(\partial_{i} \sqrt{h}(y)+\sqrt{h}(y) \frac{\partial}{\partial y^{i}}\right) \\
\bar{Q}_{0}{ }_{0}(x, y)= & -\overline{\delta(y, x)} \sqrt{h}(y)+\overline{\delta(y, x)} \frac{\pi(y) p_{\lambda}^{0}}{\kappa^{2}(y) \int d^{3} z \sqrt{h}}, \\
\bar{Q}_{i}{ }_{i}(x, y)= & \overline{\delta(y, x)}\left(\partial_{i} p_{\lambda}(y)+p_{\lambda}(y) \frac{\partial}{\partial y^{i}}\right) \\
& -\overline{\delta(y, x)} \frac{p_{\lambda}^{0}}{\int d^{3} z \sqrt{h}}\left(\partial_{i} \sqrt{h}(y)+\sqrt{h}(y) \frac{\partial}{\partial y^{i}}\right) .
\end{aligned}
$$

This completes the calculation of the full gravitational (gauge-fixed) action for quantization. The action $S+S_{\mathrm{gh}+\mathrm{gf}}$ with Eq. (3.77) admits the BRST symmetry associated with the generators $\left(\mathcal{H}_{\mu}, \overline{\mathcal{B}}, \overline{\mathcal{C}}\right)$ and the gauge conditions $\left(\chi^{\mu}, \overline{P_{\kappa^{2}}}, \overline{p_{\lambda}}\right)$.

For the chosen gauge (3.78), however, we observe that the ghost structure associated with the generators (3.79) is essentially trivial. Integration over the ghosts $\bar{b}_{m}$ and $\bar{c}^{m}(m=1,2)$ indeed gives a unit contribution to the path integral, $\operatorname{det} \overline{\mathcal{F}}_{n}^{m}=1$, since the term involving $\bar{b}_{m}$ and $c^{\mu}$ does not contribute to the result due to the lack of a term involving $b_{\mu}$ and $\bar{c}^{m}$. In other words, here the functional determinant of Eq. (3.73) factors as $\operatorname{det} \mathcal{F}_{\beta}^{\alpha}=\operatorname{det} Q^{\mu}{ }_{\nu} \times \operatorname{det} \overline{\mathcal{F}}_{n}^{m}$. Then integration over the oscillating modes $\overline{\kappa^{2}}, \overline{P_{\kappa^{2}}}, \bar{\lambda}$ and $\overline{p_{\lambda}}$ becomes trivial due to the constraints (3.78) and (3.79). The zero modes $\kappa_{0}^{2}$ and $\lambda_{0}$ remain. Hence the obtained path integral is the same as the one obtained for the reduced system in Sect. 3.4. The path integral will be discussed further in Sect. 4 . 


\section{Path integral and a relation of the gravitational and cosmological constants}

\subsection{Canonical path integral}

The canonical path integral for the gravitational sector of the local vacuum energy sequestering model is obtained as

$$
\begin{aligned}
Z_{\mathrm{VES}}=\mathcal{N}_{1} \int & \prod_{x^{\mu}} \mathcal{D} N \mathcal{D} N^{i} \mathcal{D} h_{i j} \mathcal{D} \pi^{i j} \mathcal{D} \kappa_{0}^{2} \mathcal{D} P_{\kappa^{2}}^{0} \mathcal{D} \lambda_{0} \mathcal{D} p_{\lambda}^{0} \delta\left(\chi^{\mu}\right) \operatorname{det}\left|\left\{\chi^{\mu}, \mathcal{H}_{\nu}\right\}\right| \\
& \times \exp \left[i \int d t d^{3} x\left(\partial_{t} h_{i j} \pi^{i j}+\partial_{t} \kappa_{0}^{2} P_{\kappa^{2}}^{0}+\partial_{t} \lambda_{0} p_{\lambda}^{0}-N \mathcal{H}_{T}-N^{i} \mathcal{H}_{i}\right],\right.
\end{aligned}
$$

where $\mathcal{N}_{1}$ is a normalization factor and the Hamiltonian and momentum constraints are given in (3.60) and (3.61). The same path integral can either be obtained from the formalism presented in Sect. 3.5 or it could be written for the reduced Hamiltonian system obtained in Sect. 3.4. Matter fields have been excluded for the time being. Integration over the momenta $P_{\kappa^{2}}^{0}$ and $p_{\lambda}^{0}$ gives $\delta\left(\partial_{t} \kappa_{0}^{2}\right) \delta\left(\partial_{t} \lambda_{0}\right)$. Therefore we decompose $\lambda_{0}$ and $\kappa_{0}^{2}$ to constant components and oscillating components over time as

$$
\lambda_{0}(t)=\varrho^{2} \Lambda+\overline{\lambda_{0}}(t), \quad \kappa_{0}^{2}(t)=\varrho^{2}+\overline{\kappa_{0}^{2}}(t),
$$

where $\varrho^{2}$ and $\Lambda$ are gravitational and cosmological constants, respectively, and the oscillating components satisfy: $\int d t \overline{\lambda_{0}}=0, \int d t \overline{\kappa_{0}^{2}}=0$. Integration over the momentum $\pi^{i j}$ can be performed in the standard way (see, e.g., Ref. [13]). Assuming that the path integral represents a vacuum transition amplitude for a vacuum state $\left|\varrho^{2}, \Lambda\right\rangle$ that corresponds to certain values of $\varrho^{2}$ and $\Lambda$, we obtain the path integral as

$$
\begin{aligned}
Z_{\mathrm{VES}}\left(\varrho^{2}, \Lambda\right)=\mathcal{N}_{2} \int \prod_{x^{\mu}} \mathcal{D} g_{\mu \nu} g^{00}(-g)^{-\frac{3}{2}} \delta\left(\chi^{\mu}\right) N \varrho^{6} \operatorname{det}\left|\left\{\chi^{\mu}, \mathcal{H}_{\nu}\right\}_{\pi^{i j}[h]}\right| \\
\quad \times \exp \left[i \frac{\varrho^{2}}{2} \int d^{4} x \sqrt{-g}\left(K_{i j} \mathcal{G}^{i j k l} K_{k l}+{ }^{(3)} R-2 \Lambda\right)\right],
\end{aligned}
$$

where the boundary conditions of the path integral are chosen to be consistent with the given values of $\varrho^{2}$ and $\Lambda$.

The extra factor $\varrho^{6}$ in the measure of Eq. (4.3) has not been absorbed into the normalization factor, since the value of $\varrho^{2}$ is set by the boundary conditions of the path integral. Furthermore, in the next subsection, we will consider a superposition of states with different values of the gravitational and cosmological constants, which results in an additional integration over $\kappa^{2}$ and $\Lambda$, where any additional dependence on those variables has to be taken into account. Alternatively, instead of including the factor $\varrho^{6}$ in the measure, it could be included in the operator (3.80). In the Dirac gauge, defined by $\chi^{0}=h_{i j} \pi^{i j}$ and $\chi^{i}=\partial_{j}\left(h^{\frac{1}{3}} h^{i j}\right)$, all the ghosts could be made to carry the same dimension, namely, the mass dimension $\left[b_{\mu}\right]=\left[c^{\mu}\right]=0$, by including $\varrho^{2}$ in each $Q^{i}{ }_{\mu}, i=1,2,3$, so that every component of the operator would have the mass dimension $\left[Q^{\mu}{ }_{\nu}\right]=4$. However, we shall keep the factor in the measure and consider a covariant gauge instead. 
Finally, we can transform to a covariant gauge, include matter (for simplicity without additional gauge symmetries below), and define the generating functional by including external sources $J^{\mu \nu}$ and $J_{\Psi}$ for the metric and the matter fields $\Psi$, respectively,

$$
\begin{aligned}
Z_{\mathrm{VES}}\left(\varrho^{2}, \Lambda\right)[J]= & \mathcal{N}_{2} \int \prod_{x^{\mu}} \mathcal{D} g_{\mu \nu} \mathcal{D} \eta_{\rho} \mathcal{D} b_{\sigma} \mathcal{D} c^{\sigma} \mathcal{D} \Psi g^{00}(-g)^{-\frac{3}{2}} \varrho^{6} \\
& \times \exp \left[i \int d ^ { 4 } x \left(\frac{\varrho^{2}}{2} \sqrt{-g}(R-2 \Lambda)-\eta_{\mu} \chi^{\mu}-b_{\mu} Q^{\mu}{ }_{\nu} c^{\nu}\right.\right. \\
& \left.\left.+\sqrt{-g} \mathcal{L}_{\mathrm{m}}\left(g_{\mu \nu}, \Psi\right)+g_{\mu \nu} J^{\mu \nu}+\Psi J_{\Psi}\right)\right]
\end{aligned}
$$

where we can use any covariant gauge such as, for example, the harmonic gauge

$$
\begin{aligned}
\chi^{\mu} & =\partial_{\nu}\left(\sqrt{-g} g^{\mu \nu}\right) \approx 0 \\
Q_{\nu}^{\mu}{ }^{\nu} & =\partial_{\nu}\left(\partial_{\rho}\left(\sqrt{-g} g^{\mu \nu} c^{\rho}\right)-\sqrt{-g} g^{\mu \rho} \partial_{\rho} c^{\nu}-\sqrt{-g} g^{\rho \nu} \partial_{\rho} c^{\mu}\right) .
\end{aligned}
$$

\subsection{Relation of the gravitational constant, the cosmological constant, and the energy density and pressure of matter}

Consider a vacuum state of the universe that is a superposition of states corresponding to different values of the gravitational and cosmological constants:

$$
|\Omega\rangle=\int d \varrho^{2} d \Lambda \omega\left(\varrho^{2}, \Lambda\right)\left|\varrho^{2}, \Lambda\right\rangle
$$

Now the general path integral representation of a vacuum transition amplitude can be written as

$$
Z_{\mathrm{VES}} \equiv\langle\Omega \mid \Omega\rangle=\int d \mu\left(\varrho^{2}, \Lambda\right) Z_{\mathrm{VES}}\left(\varrho^{2}, \Lambda\right)
$$

where the measure is defined by

$$
d \mu\left(\varrho^{2}, \Lambda\right)=\left|\omega\left(\varrho^{2}, \Lambda\right)\right|^{2} d \varrho^{2} d \Lambda
$$

and we assume

$$
\left\langle\varrho^{2}, \Lambda \mid \varrho^{2^{\prime}}, \Lambda^{\prime}\right\rangle=0 \text { if } \Lambda \neq \Lambda^{\prime} \text { or } \varrho^{2} \neq \varrho^{2^{\prime}} .
$$

A priori we do not know the measure (4.9) for the integration of the gravitational and cosmological constants. Therefore we assume that the measure is smooth and includes all values of $\varrho^{2} \geq 0$ and $\Lambda$. The generating functional is obtained as

$$
Z_{\mathrm{VES}}[J]=\int d \mu\left(\varrho^{2}, \Lambda\right) Z_{\mathrm{VES}}\left(\varrho^{2}, \Lambda\right)[J]
$$

This differs from the generating functional of fully diffeomorphism-invariant unimodular gravity [13] in two ways: we have an additional integration over the gravitational constant $\varrho^{2}$ and there is an additional factor $\varrho^{6}$ in the measure. Note that the latter can be absorbed into the measure (4.9) of the integral (4.11).

The question of much interest is whether we can derive a relation between the expectation values of $\varrho^{2}, \Lambda$ and the integrated matter energy density over spacetime. That would be a generalization of the result obtained in unimodular gravity [9, 10, 12, 13]. This can be done in a semiclassical approximation of the background 
field approach to the quantization of a gravitational field theory (for a review of the background field method see, e.g., Ref. [26]). The idea is that when we integrate over the metric and the matter fields, the dominant contribution comes from the configurations $\left(g_{\mu \nu}, \Psi\right)$ that solve the Einstein field equation $\left(G_{\mu \nu}+\Lambda g_{\mu \nu}=\varrho^{-2} T_{\mu \nu}\right)$ for given $\varrho^{2}$ and $\Lambda$. Then the semiclassical approximation of the path integral (4.8) becomes a sum over such configurations $\left(g_{\mu \nu}, \Psi\right)$ :

$$
Z_{\mathrm{VES}} \approx \int d \mu\left(\varrho^{2}, \Lambda\right) \sum_{\left(g_{\mu \nu}, \Psi\right)} \exp \left[i \int d^{4} x \sqrt{-g}\left(\varrho^{2} \Lambda-\frac{T}{2}+\mathcal{L}_{\mathrm{m}}\right)\right]
$$

where the trace of the Einstein equation was used to write $R=4 \Lambda-\varrho^{-2} T$. Here $\varrho^{2}$ and $\Lambda$ are the renormalized gravitational and cosmological constants. According to the stationary phase approximation the integral (4.12) is dominated by solutions for which the on-shell action vanishes. For a perfect fluid the on-shell action is given by the integral of pressure $p$ over spacetime [27], $\int d^{4} x \sqrt{-g} \mathcal{L}_{\mathrm{m}}=\int d^{4} x \sqrt{-g} p$, and the trace of the energy-momentum tensor is $T=-\rho+3 p$, where $\rho$ is the energy density. If several perfect-fluid components are considered, we have the total pressure $p=\sum_{a} p_{a}$ and the total energy density $\rho=\sum_{a} \rho_{a}$ instead. Hence we see that the most likely values of the gravitational constant and the cosmological constant are related to the average values of the total pressure and the total energy density over the whole spacetime as

$$
\varrho^{2} \Lambda \approx \frac{\int d^{4} x \sqrt{-g}\left(\frac{T}{2}-\mathcal{L}_{\mathrm{m}}\right)}{\int d^{4} x \sqrt{-g}}=\frac{\int d^{4} x \sqrt{-g}(p-\rho)}{2 \int d^{4} x \sqrt{-g}}=\frac{1}{2}\langle p-\rho\rangle .
$$

This relation approximates the product $\varrho^{2} \Lambda$ (or the ratio $\Lambda / G$ ) of the gravitational constant and the cosmological constant, but it does not tell us anything about their separate values. Hence the relation (4.13) has a very different nature compared to that obtained in unimodular gravity, although the form of the relation is similar.

Naturally, estimating the average values of the total pressure and the total energy density over the history of the universe is quite difficult, which gives a reason to doubt the usefulness of the relation (4.13). Still it is interesting that the local formulation of vacuum energy sequestering implies such a relation between the two given fundamental constants of nature.

\section{Topological induced gravity}

For linear functions $\sigma$ and $\hat{\sigma}$ the action of the local vacuum energy sequestering model (2.10) reduces to

$$
S_{\mathrm{l}}=\int d^{4} x \sqrt{-g}\left[\frac{\kappa^{2}}{2}\left(R+M_{\mathrm{P}}^{-2} \nabla_{\mu} U^{\mu}\right)-\lambda\left(1-\mu^{-4} \nabla_{\mu} V^{\mu}\right)+\mathcal{L}_{\mathrm{m}}\left(g_{\mu \nu}, \Psi\right)\right],
$$

where we have chosen $\sigma(s)=\frac{1}{2} s$ and $\hat{\sigma}(s)=s$ for simplicity. Incidentally, this action is essentially the same as the recently discussed "topological induced gravity" [20] 5 It is thus clear that the so-called topological induced gravity is the simplest special case of the local vacuum energy sequestering model. The action differs from the fully

\footnotetext{
${ }^{5}$ The scales $M_{\mathrm{P}}^{-2}$ and $\mu^{-4}$ could be absorbed into the vector fields $U^{\mu}$ and $V^{\mu}$, respectively, but that would obscure the relation to the vacuum energy sequestering theory.
} 
diffeomorphism-invariant version of unimodular gravity [13] by the presence of the vector $U^{\mu}$ and a variable gravitational coupling $\kappa^{2}$. The action can again be rewritten as in Eq. (3.1). The canonical structure is identical to that of local vacuum energy sequestering obtained in Sect. 3 with the substitutions $\sigma^{\prime}=\frac{1}{2}$ and $\hat{\sigma}^{\prime}=1$.

The action (5.1) can be written in a BRST-exact form by including an appropriate ghost action [20]. This justifies the label "topological". It is natural to ask whether such a BRST-exact formulation can be generalized to the local theory of vacuum energy sequestering with nonlinear functions $\sigma$ and $\hat{\sigma}$. This will be explored next.

\section{Induced theory of vacuum energy sequestering from gauge fixing}

\subsection{Gravitational action from gauge fixing}

Here we consider a BRST-exact formulation for the local version of vacuum energy sequestering. The gravitational action (2.10) will be seen to emerge as a gauge-fixing action. The approach is similar to those of Refs. [20] and [21].

Consider a theory for two vector fields $U^{\mu}$ and $V^{\mu}$ on a curved spacetime with metric $g_{\mu \nu}$, and optionally some matter fields $\Psi$. The theory is assumed to be diffeomorphism invariant. The gravitational action of the theory is assumed to vanish initially, i.e., we consider an action of the form

$$
S=S_{\mathrm{v}}\left[g_{\mu \nu}, U^{\mu}, V^{\nu}\right]+S_{\mathrm{m}}\left[g_{\mu \nu}, \Psi\right] .
$$

Including couplings between matter fields $\Psi$ and the vector fields $U^{\mu}$ and $V^{\mu}$ would be possible as well. Hence we have no theory of gravity in the beginning. We assume that the action for the vector fields possesses a gauge symmetry under transformations of $U^{\mu}$ and $V^{\mu}$. In the simplest case, one could consider a vanishing action, $S_{\mathrm{v}}=0$, so that the action would be invariant under any transformation of $U^{\mu}$ and $V^{\mu}$. Here we consider that the action $S_{\mathrm{v}}$ is invariant under the following two gauge transformations generated by infinitesimal scalar field parameters $\alpha$ and $\hat{\alpha}$ with zero mass dimension:

$$
\delta_{\alpha} U^{\mu}=M_{\mathrm{P}}^{2} \nabla^{\mu} \alpha, \quad \delta_{\hat{\alpha}} V^{\mu}=\mu^{2} \nabla^{\mu} \hat{\alpha} .
$$

The gauge-fixing action for $U^{\mu}$ and $V^{\mu}$ can be chosen as the gravitational part of the local vacuum energy sequestering action (2.10),

$$
S_{\mathrm{gf}}=\int d^{4} x \sqrt{-g}\left[\frac{\kappa^{2}}{2} R-\lambda+\sigma\left(\frac{\kappa^{2}}{M_{\mathrm{P}}^{2}}\right) \nabla_{\mu} U^{\mu}+\hat{\sigma}\left(\frac{\lambda}{\mu^{4}}\right) \nabla_{\mu} V^{\mu}\right],
$$

where $\kappa^{2}$ and $\lambda$ are the auxiliary scalar fields required for imposing the gauge-fixing conditions for the gauge symmetry under Eq. (6.2). The gauge conditions are written as

$$
\sigma^{\prime}\left(\frac{\kappa^{2}}{M_{\mathrm{P}}^{2}}\right) \nabla_{\mu} U^{\mu}+\frac{M_{\mathrm{P}}^{2}}{2} R=0, \quad \hat{\sigma}^{\prime}\left(\frac{\lambda}{\mu^{4}}\right) \nabla_{\mu} V^{\mu}-\mu^{4}=0 .
$$

Note that the auxiliary variables $\kappa^{2}$ and $\lambda$ appear in the gauge conditions (6.4) when the functions $\sigma$ and $\hat{\sigma}$ are nonlinear, since then the gauge-fixing Lagrangian (6.3) is not linear in the auxiliary fields, which is a rather uncommon situation.

We have chosen the gauge-fixing action (6.3) to specifically match the action of the local formulation of vacuum energy sequestering. However, the gauge-fixing action 
could be chosen in a number of different ways. There are three kinds of changes that could be considered. First, the curvature part of the action (6.3) could be changed. For example, the gravitational part of the action could be defined to include higher-order curvature terms, but that would require additional coupling constants or fields, unless the coefficients of the higher-curvature terms are set to $\pm \kappa^{2} M_{\mathrm{P}}^{-n}, n=2,4, \ldots$, which would give weak couplings that are preferable regarding long-distance behavior 6 In principle, we could even abandon full diffeomorphism invariance and use, for example, the Lagrangian density of Hořava-Lifshitz gravity [29] (with a variable $\kappa^{2}$ ) in place of the Einstein-Hilbert term, $\frac{\kappa^{2}}{2} \sqrt{-g} R$. Second, the part of the gauge-fixing action (6.3) that involves $U^{\mu}$ and $V^{\mu}$ could be chosen differently, e.g. to include quadratic terms $U_{\mu} U^{\mu}$ or direct coupling to curvature etc. That kind of modification, however, could lead to a completely different type of theory, since we would no longer obtain the field equations $\nabla_{\mu} \kappa^{2}=0$ and $\nabla_{\mu} \lambda=0$; the same happens when a nonvanishing action $S_{\mathrm{v}}$ for the vector fields is included. Finally, we could consider different gauge symmetry transformations instead of Eq. (6.2). Above we have considered the simplest gauge transformations that imply scalar ghost fields. The gauge transformations could be parametrized by vector fields or tensor fields of higher rank, which would require vector ghost fields or higher-rank tensor ghost fields, respectively. In summary, the approach explored here can be used to construct a wide variety of gravitational models with variable gravitational couplings and a variable cosmological (constant) parameter.

In order to obtain a BRST-invariant (and eventually BRST-exact) action, we introduce Grassman-odd ghost fields $c, \hat{c}$ and antighost fields $b, \hat{b}$. The two BRST transformations can be obtained from the gauge transformations (6.2) as

$$
\begin{array}{ccc}
\delta_{B} \kappa^{2}=0, & \delta_{B} U^{\mu}=\epsilon M_{\mathrm{P}}^{2} \nabla^{\mu} c, \quad \delta_{B} c=0, & \delta_{B} b=\epsilon \sigma\left(\frac{\kappa^{2}}{M_{\mathrm{P}}^{2}}\right), \\
\hat{\delta}_{B} \lambda=0, & \hat{\delta}_{B} V^{\mu}=\hat{\epsilon} \mu^{2} \nabla^{\mu} \hat{c}, \quad \hat{\delta}_{B} \hat{c}=0, \quad \hat{\delta}_{B} \hat{b}=\hat{\epsilon} \hat{\sigma}\left(\frac{\lambda}{\mu^{4}}\right),
\end{array}
$$

where $\epsilon$ and $\hat{\epsilon}$ are infinitesimal anticommuting parameters. Note that the gauge transformations (6.2) are Abelian so that the BRST transformations of the ghosts $(c, \hat{c})$ vanish. The BRST transformations of the antighosts $(b, \hat{b})$ are nonlinear in the auxiliary fields due to the corresponding nonlinearity of the gauge-fixing Lagrangian (6.3). The ghost action can be obtained as

$$
\begin{aligned}
S_{\mathrm{gh}} & =-\int d^{4} x \sqrt{-g}\left(b M_{\mathrm{P}}^{2} \nabla_{\mu} \nabla^{\mu} c+\hat{b} \mu^{2} \nabla_{\mu} \nabla^{\mu} \hat{c}\right) \\
& =\int d^{4} x \sqrt{-g}\left(M_{\mathrm{P}}^{2} \nabla_{\mu} b \nabla^{\mu} c+\mu^{2} \nabla_{\mu} \hat{b} \nabla^{\mu} \hat{c}\right)
\end{aligned}
$$

The gravitational action, $S_{g}=S_{\mathrm{gf}}+S_{\mathrm{gh}}$, is BRST invariant under Eqs. (6.5) and (6.6)).

\footnotetext{
${ }^{6}$ One of the candidates for a modification of the gravitational sector would be Weyl gravity, due to its known structure and relevance. However, as we have discussed in the last paragraph of Sect. 2.1. the smallness of the cosmological constant in the sequestering mechanism is due to two approximate symmetries, namely, scaling and shift symmetries, which are broken by the gravitational sector. Thus, since pure Weyl gravity is scale invariant, there would be no gravitational scale $\kappa^{2}$, but rather a dimensionless gravitational coupling. Furthermore, the cosmological constant term could not be included in Weyl gravity, since it ruins the canonical and geometric structure due to the appearance of a constraint $\sqrt{-g}=0$ [28]. Extending the action (6.3) with the conformally invariant Weyl action would not imply such problems, since then the approximate scaling and shift symmetries persist, and the canonical structure of the Einstein-Hilbert plus Weyl action is known to be consistent [28].
} 
The gravitational part of the action is also BRST exact, since it can be written as

$$
S_{g}=\int d^{4} x \sqrt{-g}\left(s_{B}\left[b\left(\frac{\kappa^{2}}{2 \sigma\left(\frac{\kappa^{2}}{M_{\mathrm{P}}^{2}}\right)} R+\nabla_{\mu} U^{\mu}\right)\right]-\hat{s}_{B}\left[\hat{b}\left(\frac{\lambda}{\hat{\sigma}\left(\frac{\lambda}{\mu^{4}}\right)}-\nabla_{\mu} V^{\mu}\right)\right]\right),
$$

where $s_{B}$ and $\hat{s}_{B}$ are the Slavnov variations corresponding to Eqs. (6.5) and (6.6), respectively. Hence it can be considered as a topological field theory [22]. The full gauge-fixed action is now given as $S=S_{g}+S_{\mathrm{v}}+S_{\mathrm{m}}$.

We should remark that the BRST-exact formulation of local vacuum energy sequestering should not be confused with the gauging procedure followed in Sect. 2.2. The main conceptual difference between the approaches is the way the gauge symmetry is imposed: the local formulation of vacuum energy sequestering is obtained by gauging the global sequestering mechanism, while here we assume a further gauge symmetry in order to obtain a BRST-exact form of the action. The present approach can be seen as an extension of the local formulation of vacuum energy sequestering.

\subsection{Hamiltonian analysis}

Our goal is to find a canonical formulation of the full gauge-fixed action $S_{g}=S_{\mathrm{gf}}+S_{\mathrm{gh}}$, where $S_{\text {gf }}$ is defined in Eq. (6.3) and $S_{\text {gf }}$ is defined in (6.7). Note that the canonical analysis of $S_{\mathrm{gf}}$ has been done in the previous section, so that we focus on the ghost action $S_{\mathrm{gh}}$. To begin with we rewrite it in the $3+1$ formalism,

$$
\begin{aligned}
S_{\mathrm{gh}}= & M_{\mathrm{P}}^{2} \int d t d^{3} x \sqrt{h} N\left(-\nabla_{n} b \nabla_{n} c+h^{i j} \partial_{i} b \partial_{j} c\right) \\
& +\mu^{2} \int d t d^{3} x \sqrt{h} N\left(-\nabla_{n} \hat{b} \nabla_{n} \hat{c}+h^{i j} \partial_{i} b \partial_{j} c\right),
\end{aligned}
$$

so that we have conjugate momenta

$$
\begin{gathered}
p_{b}=\frac{\delta \mathcal{L}_{g h}}{\delta^{L} \partial_{t} b}=-M_{\mathrm{P}}^{2} \sqrt{h} \nabla_{n} c, \quad p_{c}=\frac{\delta \mathcal{L}_{g h}}{\delta^{L} \partial_{t} c}=M_{\mathrm{P}}^{2} \sqrt{h} \nabla_{n} b, \\
p_{\hat{b}}=\frac{\delta \mathcal{L}_{g h}}{\delta^{L} \partial_{t} \hat{b}}=-\mu^{2} \sqrt{h} \nabla_{n} \hat{c}, \quad p_{\hat{c}}=\frac{\delta \mathcal{L}_{g h}}{\delta^{L} \partial_{t} \hat{c}}=\mu^{2} \sqrt{h} \nabla_{n} \hat{b}
\end{gathered}
$$

with the following nonvanishing (graded) Poisson brackets:

$$
\begin{aligned}
\left\{c(x), p_{c}(y)\right\} & =\left\{p_{c}(y), c(x)\right\}-\delta(x-y), \\
\left\{b(x), p_{b}(y)\right\} & =\left\{p_{b}(y), b(x)\right\}=-\delta(x-y), \\
\left\{\hat{c}(x), p_{\hat{c}}(y)\right\} & =\left\{p_{\hat{c}}(y), \hat{c}(x)\right\}=-\delta(x-y), \\
\left\{\hat{b}(x), p_{\hat{b}}(y)\right\} & =\left\{p_{\hat{b}}(y), \hat{b}(x)\right\}=-\delta(x-y) .
\end{aligned}
$$

Then it is easy to find the ghost contributions to the Hamiltonian and diffeomorphism constraints as

$$
\begin{aligned}
\mathcal{H}_{T}^{\mathrm{gh}} & =\frac{1}{M_{\mathrm{P}}^{2} \sqrt{h}} p_{c} p_{b}-M_{P}^{2} \sqrt{h} h^{i j} \partial_{i} b \partial_{j} c+\frac{1}{\mu^{2} \sqrt{h}} p_{\hat{c}} p_{\hat{b}}-\mu^{2} \sqrt{h} h^{i j} \partial_{i} \hat{b} \partial_{j} \hat{c} \\
\mathcal{H}_{i}^{\mathrm{gh}} & =\partial_{i} c p_{c}+\partial_{i} b p_{b}+\partial_{i} \hat{c} p_{\hat{c}}+\partial_{i} \hat{b} p_{\hat{b}} .
\end{aligned}
$$


Using the standard Noether method, we derive the conserved BRST currents as

$$
\begin{aligned}
& J_{B R S T}^{\mu}=\sqrt{-g} M_{\mathrm{P}}^{2} \sigma\left(\frac{\kappa^{2}}{M_{\mathrm{P}}^{2}}\right) g^{\mu \nu} \nabla_{\nu} c, \\
& \hat{J}_{B R S T}^{\mu}=\sqrt{-g} \mu^{2} \hat{\sigma}\left(\frac{\lambda}{\mu^{2}}\right) g^{\mu \nu} \nabla_{\nu} \hat{c},
\end{aligned}
$$

and hence we have corresponding conserved charges expressed in terms of the canonical variables,

$$
\begin{aligned}
Q_{B R S T} & =\int d^{3} x \sigma\left(\frac{\kappa^{2}}{M_{\mathrm{P}}^{2}}\right) p_{b}, \\
\hat{Q}_{B R S T} & =\int d^{3} x \hat{\sigma}\left(\frac{\lambda}{\mu^{2}}\right) p_{\hat{b}} .
\end{aligned}
$$

Since we want these charges to act nontrivially on $U^{\mu}$ and $V^{\mu}$, respectively, we add to them linear combinations of the constraints $P_{\boldsymbol{n}}, P_{i}$ and $p_{\boldsymbol{n}}, p_{i}$, respectively. In fact, note that (6.2) implies

$$
\delta_{\alpha} U_{n}=M_{P}^{2} \nabla_{n} \alpha, \quad \delta_{\alpha} U^{i} M_{P}^{2} h^{i j} \partial_{j} \alpha
$$

and also

$$
\delta_{\hat{\alpha}} V_{\boldsymbol{n}}=\mu^{2} \nabla_{n} \hat{\alpha}, \quad \delta_{\hat{\alpha}} V^{i}=\mu^{2} h^{i j} \nabla_{j} \hat{\alpha} .
$$

From these transformation rules we deduce the BRST transformations

$$
\delta_{B} U_{\boldsymbol{n}}=-\epsilon \frac{1}{\sqrt{h}} p_{b}, \quad \delta_{B} U^{i}=\epsilon M_{P}^{2} h^{i j} \partial_{j} c
$$

and hence we find that the extended BRST operator has the form

$$
Q_{B R S T}=\int d^{3} x\left(\sigma\left(\frac{\kappa^{2}}{M_{\mathrm{P}}^{2}}\right) p_{b}-\frac{p_{b}}{\sqrt{h}} P_{\boldsymbol{n}}+M_{P}^{2} P_{i} h^{i j} \partial_{j} c\right) .
$$

In the same way, we write an extended form of the BRST operator $\hat{Q}_{B R S T}$ as

$$
\hat{Q}_{B R S T}=\int d^{3} x\left(\hat{\sigma}\left(\frac{\lambda}{\mu^{2}}\right) p_{\hat{b}}-\frac{p_{\hat{b}}}{\sqrt{h}} p_{\boldsymbol{n}}+\mu^{2} p_{i} h^{i j} \partial_{j} \hat{c}\right) .
$$

The BRST charges have the following nonvanishing Poisson brackets with the canonical variables:

$$
\begin{aligned}
\left\{Q_{B R S T}, b\right\} & =-\sigma\left(\frac{\kappa^{2}}{M_{\mathrm{P}}^{2}}\right), \quad\left\{Q_{B R S T}, P_{\kappa^{2}}\right\}=\frac{1}{M_{\mathrm{P}}^{2}} \sigma^{\prime}\left(\frac{\kappa^{2}}{M_{\mathrm{P}}^{2}}\right) p_{b}, \\
\left\{Q_{B R S T}, U_{\boldsymbol{n}}\right\} & =\frac{1}{\sqrt{h}} p_{b}, \quad\left\{Q_{B R S T}, U^{i}\right\}=-M_{P}^{2} h^{i j} \partial_{j} c, \\
\left\{\hat{Q}_{B R S T}, \hat{b}\right\} & =-\hat{\sigma}\left(\frac{\lambda}{\mu^{2}}\right), \quad\left\{\hat{Q}_{B R S T}, p_{\lambda}\right\}=\frac{1}{\mu^{2}} \hat{\sigma}^{\prime}\left(\frac{\lambda}{\mu^{2}}\right) p_{\hat{b}}, \\
\left\{\hat{Q}_{B R S T}, V_{\boldsymbol{n}}\right\} & =\frac{1}{\sqrt{h}} p_{\hat{b}}, \quad\left\{\hat{Q}_{B R S T}, V^{i}\right\}=-\mu^{2} h^{i j} \partial_{j} \hat{c} .
\end{aligned}
$$

The BRST charges (6.20) and (6.21) Poisson commute with the second-class constraints (3.5) and (3.22), which explains why the momenta $P_{\kappa^{2}}$ and $p_{\lambda}$ must have 
nonvanishing BRST transformations (6.22), while the conjugated variables $\kappa^{2}$ and $\lambda$ do not change under the given BRST transformations.

The Poisson brackets between the BRST charges and the Hamiltonian constraint are now now easily obtained as

$$
\begin{aligned}
& \left\{Q_{B R S T}, \mathcal{H}_{T}^{\prime \prime}+\mathcal{H}_{T}^{\text {ghost }}\right\} \approx \sigma^{\prime} \sqrt{h} h^{i j} \partial_{i} \kappa^{2} \partial_{j} c=\sigma^{\prime} \sqrt{h} h^{i j} \mathcal{B}_{i} \partial_{j} c \approx 0, \\
& \left\{\hat{Q}_{B R S T}, \mathcal{H}_{T}^{\prime \prime}+\mathcal{H}_{T}^{\text {ghost }}\right\} \approx \hat{\sigma}^{\prime} \sqrt{h} h^{i j} \partial_{i} \lambda \partial_{j} \hat{c}=\hat{\sigma}^{\prime} \sqrt{h} h^{i j} \mathcal{C}_{i} \partial_{j} \hat{c} \approx 0,
\end{aligned}
$$

up to terms proportional to the constraints $P_{\boldsymbol{n}} \approx 0$ and $p_{\boldsymbol{n}} \approx 0$. The BRST charges have vanishing Poisson brackets with all the other constraints. Then it is easy to derive the following relation

$$
\left\{Q_{B R S T},-\frac{b}{\sigma} \mathcal{H}_{T}^{\prime \prime}\right\}=\mathcal{H}_{T}^{\prime \prime}-b \frac{\sigma^{\prime}}{\sigma} \mathcal{B}_{i} h^{i j} \sqrt{h} \partial_{j} c
$$

Then we could replace $\mathcal{H}_{T}^{\prime \prime}$ in the definition of the Hamiltonian with this Poisson bracket when the expression proportional to $\mathcal{B}_{i}$ is absorbed into the corresponding Lagrange multiplier. In other words, we see that the Hamiltonian has the schematic form

$$
H=\left\{Q_{B R S T}, \Psi\right\}+\left\{\hat{Q}_{B R S T}, \hat{\Psi}\right\},
$$

where the explicit form of the gauge-fixing fermions $\Psi$ and $\hat{\Psi}$ is not important for us. On the other hand, the fact that the Hamiltonian can be written in the form (6.26) is a consequence of the fact that the gravitational action $S_{\mathrm{g}}(\underline{6.8})$ is BRST exact.

\section{A simple model for including the cosmological constant as a topological field theory}

Let us consider the model proposed in Ref. [21]. In this model, the cosmological constant part of the Lagrangian is BRST exact, while the Einstein-Hilbert gravitational part appears as usual. In other words, this model is an extension of unimodular gravity in the same way that our formulation in Sect. 6 extends local vacuum energy sequestering. In the model of Ref. [21], however, only the cosmological constant part of the action is made topological, while in Sect. 6 the whole gravitational action of the local version of vacuum energy sequestering was formulated as a topological field theory. We should note that the model of Ref. [21] is unlikely to actually solve any cosmological constant problem, since it lacks a mechanism for ensuring the perturbative stability of the cosmological constant. In this respect, the model is on par with conventional unimodular gravity. Nevertheless, the model is an interesting example for building a topological field theory for the cosmological constant.

The model is defined by the action

$$
S=\int d^{4} x \sqrt{-g}\left(\frac{M_{\mathrm{P}}^{2}}{2} R-\lambda+\frac{1}{\mu^{3}} \partial_{\mu} \lambda g^{\mu \nu} \partial_{\nu} \phi-\partial_{\mu} b g^{\mu \nu} \partial_{\nu} c\right),
$$

where $\phi$ is a scalar field and the fields $b$ and $c$ are Grassmann-odd ghosts. The action is invariant under BRST transformation

$$
\delta \lambda=\delta c=0, \quad \delta \phi=\epsilon c, \quad \delta b=\frac{1}{\mu^{3}} \epsilon \lambda
$$


where $\epsilon$ is a global fermionic (anticommuting) parameter. Note that this BRST transformation implies the existence of BRST current $j_{B R S T}^{\mu}$ in the form

$$
j_{B R S T}^{\mu}=\frac{1}{\mu^{3}} \sqrt{-g}\left(-c g^{\mu \nu} \partial_{\nu} \lambda+g^{\mu \nu} \partial_{\nu} c \lambda\right), \quad \partial_{\mu} j_{B R S T}^{\mu}=0 .
$$

As a result we have a conserved charge

$$
Q_{B R S T}=\int d^{3} x j^{0}=\frac{1}{\mu^{3}} \int d^{3} x\left(-c \sqrt{-g} g^{0 \nu} \partial_{\nu} \lambda+\sqrt{-g} g^{0 \nu} \partial_{\nu} c \lambda\right) .
$$

Our goal is to proceed to the Hamiltonian formalism. Note that in the $3+1$ formalism the action has the form

$$
\begin{array}{r}
S=\int d^{4} x N \sqrt{h}\left(\frac{M_{\mathrm{P}}^{2}}{2}\left(K_{i j} \mathcal{G}^{i j k l} K_{k l}+{ }^{(3)} R\right)-\lambda-\frac{1}{\mu^{3}} \nabla_{n} \lambda \nabla_{n} \phi+\frac{1}{\mu^{3}} \partial_{i} \lambda h^{i j} \partial_{j} \phi\right. \\
\left.+\nabla_{n} b \nabla_{n} c-h^{i j} \partial_{i} b \partial_{j} c\right) .
\end{array}
$$

In the same way as in previous sections we obtain

$$
\pi^{i j}=\frac{M_{\mathrm{P}}^{2}}{2} \mathcal{G}^{i j k l} K_{k l}, \quad \pi_{N} \approx 0, \quad \pi_{i} \approx 0, \quad p_{\lambda}=-\frac{\sqrt{h}}{\mu^{3}} \nabla_{n} \phi, \quad p_{\phi}=-\frac{\sqrt{h}}{\mu^{3}} \nabla_{n} \lambda .
$$

In case of the Grassman-odd variables we have to be careful with the definition of the momenta. We define them in terms of the variation from the left as

$$
p_{b}=\sqrt{h} \nabla_{n} c, \quad p_{c}=-\sqrt{h} \nabla_{n} b,
$$

so that the Hamiltonian is equal to

$$
\begin{aligned}
H= & \int d^{3} x\left(N \mathcal{H}_{T}+N^{i} \mathcal{H}_{i}\right) \\
\mathcal{H}_{T}= & \frac{2}{M_{\mathrm{P}}^{2} \sqrt{h}} \pi^{i j} \mathcal{G}_{i j k l} \pi^{k l}-\frac{\mu^{3}}{\sqrt{h}} p_{\phi} p_{\lambda}-\frac{1}{\sqrt{h}} p_{c} p_{b}- \\
& -\frac{M_{\mathrm{P}}^{2}}{2} \sqrt{h^{(3)}} R+\sqrt{h} \lambda-\frac{1}{\mu^{3}} \sqrt{h} \partial_{i} \lambda h^{i j} \partial_{j} \phi+\sqrt{h} h^{i j} \partial_{i} b \partial_{j} c \\
\mathcal{H}_{i}= & -2 h_{i k} D_{i} \pi^{i k}+\partial_{i} c p_{c}+\partial_{i} b p_{b}+\partial_{i} \lambda p_{\lambda}+\partial_{i} \phi p_{\phi} .
\end{aligned}
$$

The BRST charge takes the form

$$
Q_{B R S T}=\int d^{3} x\left(-c p_{\phi}-\frac{1}{\mu^{3}} p_{b} \lambda\right)
$$

so that

$$
\begin{gathered}
\left\{Q_{B R S T}, \phi\right\}=c, \quad\left\{Q_{B R S T}, b\right\}=\frac{1}{\mu^{3}} \lambda, \\
\left\{Q_{B R S T}, p_{c}\right\}=p_{\phi}, \quad\left\{Q_{B R S T}, p_{\lambda}\right\}=-\frac{1}{\mu^{3}} p_{b},
\end{gathered}
$$

using also the fact that $b, p_{b}$ and $c, p_{c}$ have graded Poisson brackets

$$
\begin{aligned}
& \left\{c(x), p_{c}(y)\right\}=\left\{p_{c}(y), c(x)\right\}=-\delta(x-y), \\
& \left\{b(x), p_{b}(y)\right\}=\left\{p_{b}(y), b(x)\right\}=-\delta(x-y) .
\end{aligned}
$$


Then it is easy to see that

$$
\left\{Q_{B R S T}, \mathcal{H}_{T}\right\}=0, \quad\left\{Q_{B R S T}, \mathcal{H}_{i}\right\}=0
$$

which is the reflection of the fact that $Q_{B R S T}$ is conserved. The question is whether the existence of this charge can remove negative unphysical states. Let us consider a phase-space function $Z$ with Grassmann parity $|Z|$. Then using the generalized Jacobi identity

$$
\{X,\{Y, Z\}\}=\{\{X, Y\}, Z\}+(-1)^{|X||Y|}\{Y,\{X, Z\}\} .
$$

Now since it is a phase-space function its Poisson bracket has to weakly vanish on the constraint surface

$$
\left\{Z, \mathcal{C}_{A}\right\}=u_{A}^{B} \mathcal{C}_{B}
$$

where $\mathcal{C}_{A}=\left(\mathcal{H}_{T}, \mathcal{H}_{i}, \pi_{N}, \pi_{i}\right)$. Note that since $Z$ has grading $|Z|$ and the constraints have $\left|\mathcal{C}_{A}\right|=0, u_{A}^{B}$ has to have the same grading as $Z$. Let us use the graded Jacobi identity above for $X=Q_{B R S T}$ and $Y=\mathcal{C}_{A}$ as

$$
\left\{Q_{B R S T},\left\{\mathcal{C}_{A}, Z\right\}\right\}=\left\{\left\{Q_{B R S T}, \mathcal{C}_{A}\right\}, Z\right\}+\left\{\mathcal{C}_{A},\left\{Q_{B R S T}, Z\right\}\right\} .
$$

Using the fact that the first expression on the right-hand side is equal to zero, we obtain

$$
\left\{Q_{B R S T}, u_{A}^{B} \mathcal{C}_{B}\right\}=\left\{\mathcal{C}_{A},\left\{Q_{B R S T}, Z\right\}\right\}
$$

Since $\left\{Q_{B R S T}, \mathcal{C}_{A}\right\}=0$, we obtain

$$
\left\{\mathcal{C}_{A},\left\{Q_{B R S T}, Z\right\}\right\}=0
$$

which implies that

$$
\left\{Q_{B R S T}, Z\right\}=v^{A} \mathcal{C}_{A}
$$

In other words, whenever $Z$ is a physical variable that is invariant under diffeomorphism, it has to have a weakly vanishing Poisson bracket with $Q_{B R S T}$.

\section{Conclusions}

We have studied the Hamiltonian formalism and path integral quantization of the local version of vacuum energy sequestering. The path integral (4.4) has a similar form as that of GR but with the values of the cosmological and gravitational constants, $\Lambda$ and $\varrho^{2}$, specified by the boundary conditions of the path integral (chosen to match their observed net values). This result is in agreement with the canonical counting of physical degrees of freedom: two propagating physical degrees of freedom for the graviton and two zero modes. The zero modes are the gravitational and cosmological constants.

Similar to the situation of unimodular gravity [13], the local formulation of vacuum energy sequestering also involves linearly dependent generators, namely, the constraints (3.30) and (3.38) that are associated with the cosmological and gravitational constants, respectively. A proper treatment of quantization and gauge invariance for a system with linearly dependent generators is achieved by means of the BatalinVilkovisky formalism [25]. This was achieved in Sect. 3.5, where we also showed that the path integral obtained via the Batalin-Vilkovisky formalism matches that of the reduced system obtained in Sect. 3.4 by eliminating several unphysical variables. 
Another similarity with unimodular gravity is the possibility of generalizing the path integral expression in order to encompass different vacuum states, which correspond to different values of the gravitational and cosmological constants. In the context of unimodular gravity, this approach leads to the so-called Ng-van Dam form for the path integral, where an additional integration over the cosmological constant is present. We have extended the idea for local vacuum energy sequestering by considering a superposition of vacuum states related to different values of the gravitational and cosmological constants. This results in a path integral with additional integration over both the gravitational constant and the cosmological constant (4.11). The most likely values of the gravitational and cosmological constants are regarded to contribute most to the path integral. Then, in the semiclassical and stationary phase approximation, the path integral implies a relation among the product $\varrho^{2} \Lambda$ (or the ratio $\Lambda / G$ ) of the gravitational constant and the cosmological constant to the average values of the total pressure and the total energy density over the whole spacetime (4.13).

For completeness, we also considered the local vacuum energy sequestering model from a new perspective of a topological or BRST-exact formulation. In this approach, the gravitational action of vacuum energy sequestering appears as a gauge-fixing action along with an appropriate ghost action. As a result, the action of vacuum energy sequestering becomes BRST exact and can be viewed as a topological field theory [Eq. (6.8)]. The topological approach was supplemented by a canonical analysis of the ghost sector, which is required in order to establish the full BRST formalism.

Finally, we remark that the vacuum energy sequestering mechanism is quite robust in its local form, and it could prove to be useful beyond Einstein gravity. We noted the possibility of generalizing or modifying the gravitational sector of the theory in a number of different ways. For example, in order to achieve a power-counting renormalizable gravitational sector, the Einstein-Hilbert part of the action could be replaced with an action of the Hořava-Lifshitz type (with a variable gravitational constant $\kappa^{2}$ ), which would be invariant under foliation-preserving diffeomorphisms. The possibility of modifying the terms that involve the auxiliary fields, or the gauge conditions imposed on the auxiliary fields, was also discussed within the BRST-exact formulation in Sect. 5. The latter could modify the sequestering mechanism drastically, and hence it should be considered cautiously.

\section{Acknowledgements}

M.O. gratefully acknowledges support from the Emil Aaltonen Foundation. R.B. thankfully acknowledges CAPES/PNPD for support, Project No. 23038007041201166. The work of J.K. was supported by the Grant Agency of the Czech Republic under the grant P201/12/G028.

\section{References}

[1] S. Weinberg, "The Cosmological Constant Problem," Rev. Mod. Phys. 61 (1989) 1 doi:10.1103/RevModPhys.61.1.

[2] A. Padilla, "Lectures on the Cosmological Constant Problem," arXiv:1502.05296 [hep-th] 
[3] A. Einstein, "Do gravitational fields play an essential part in the structure of the elementary particles of matter?," Sitzungsber. Preuss. Akad. Wiss. Berlin (Math. Phys.) 1919 (1919) 433, translated and included in The Principle of Relativity, by H.A. Lorentz et al. (Dover Press, New York, 1923).

[4] J. J. van der Bij, H. van Dam and Y. J. Ng, "The Exchange of Massless Spin Two Particles," Physica 116A (1982) 307. doi:10.1016/0378-4371(82)90247-3

[5] W. Buchmuller and N. Dragon, "Einstein Gravity From Restricted Coordinate Invariance," Phys. Lett. B 207 (1988) 292. doi:10.1016/0370-2693(88)90577-1

[6] A. Padilla and I. D. Saltas, "A note on classical and quantum unimodular gravity," Eur. Phys. J. C 75 (2015) 561 doi:10.1140/epjc/s10052-015-3767-0 arXiv:1409.3573 [gr-qc]].

[7] W. G. Unruh, "A Unimodular Theory of Canonical Quantum Gravity," Phys. Rev. D 40 (1989) 1048. doi:10.1103/PhysRevD.40.1048

[8] M. Henneaux and C. Teitelboim, "The Cosmological Constant and General Covariance," Phys. Lett. B 222 (1989) 195 doi:10.1016/0370-2693(89)91251-3.

[9] Y. J. Ng and H. van Dam, "Possible solution to the cosmological constant problem," Phys. Rev. Lett. 65 (1990) 1972 doi:10.1103/PhysRevLett.65.1972.

[10] Y. J. Ng and H. van Dam, "Unimodular theory of gravity and the cosmological constant," J. Math. Phys. 32 (1991) 1337 doi:10.1063/1.529283.

[11] K. V. Kuchar, "Does an unspecified cosmological constant solve the problem of time in quantum gravity?," Phys. Rev. D 43 (1991) 3332. doi:10.1103/PhysRevD.43.3332.

[12] L. Smolin, "Quantization of unimodular gravity and the cosmological constant problems," Phys. Rev. D 80 (2009) 084003 doi:10.1103/PhysRevD.80.084003 arXiv:0904.4841 [hep-th]].

[13] R. Bufalo, M. Oksanen and A. Tureanu, "How unimodular gravity theories differ from general relativity at quantum level," Eur. Phys. J. C 75 (2015) 477 doi:10.1140/epjc/s10052-015-3683-3 arXiv:1505.04978 [hep-th]].

[14] N. Kaloper and A. Padilla, "Sequestering the Standard Model Vacuum Energy," Phys. Rev. Lett. 112 (2014) 091304 doi:10.1103/PhysRevLett.112.091304 arXiv:1309.6562 [hep-th]].

[15] N. Kaloper and A. Padilla, "Vacuum Energy Sequestering: The Framework and Its Cosmological Consequences," Phys. Rev. D 90 (2014) 084023 doi:10.1103/PhysRevD.90.084023 [Phys. Rev. D 90 (2014) 109901 doi:10.1103/PhysRevD.90.109901] [arXiv:1406.0711 [hep-th]].

[16] N. Kaloper and A. Padilla, "Sequestration of Vacuum Energy and the End of the Universe," Phys. Rev. Lett. 114 (2015) 101302 doi:10.1103/PhysRevLett.114.101302 arXiv:1409.7073 [hep-th]].

[17] C. Lanczos, The Variational Principles of Mechanics, 4th ed. (University of Toronto Press, Toronto, 1970). 
[18] N. Kaloper, A. Padilla, D. Stefanyszyn and G. Zahariade, "A Manifestly Local Theory of Vacuum Energy Sequestering," Phys. Rev. Lett. 116 (2016) 051302 doi:10.1103/PhysRevLett.116.051302 arXiv:1505.01492 [hep-th].

[19] N. Kaloper, A. Padilla and D. Stefanyszyn, "Sequestering effects on and of vacuum decay," Phys. Rev. D 94 (2016) 025022 doi:10.1103/PhysRevD.94.025022 arXiv:1604.04000 [hep-th].

[20] I. Oda, "Induced Gravity and Topological Quantum Field Theory," arXiv:1602.03478 [gr-qc]; "Topological Induced Gravity," arXiv:1603.00112 [grqc].

[21] S. Nojiri, "A simple solution for one of the cosmological constant problems," arXiv:1601.02203 [hep-th].

[22] E. Witten, "Topological Quantum Field Theory," Commun. Math. Phys. 117 (1988) 353 doi:10.1007/BF01223371.

[23] P. A. M. Dirac, Lectures on Quantum Mechanics, (Yeshiva University, New York, 1964).

[24] J. Klusoň, "Note about canonical formalism for normalized gravity and vacuum energy sequestering model," JHEP 1501 (2015) 085 doi:10.1007/JHEP01(2015)085 arXiv:1411.7501 [hep-th]].

[25] I. A. Batalin and G. A. Vilkovisky, "Quantization of Gauge Theories with Linearly Dependent Generators," Phys. Rev. D 28 (1983) 2567 doi:10.1103/PhysRevD.28.2567.

[26] S. L. Adler, "Einstein Gravity as a Symmetry Breaking Effect in Quantum Field Theory," Rev. Mod. Phys. 54 (1982) 729 doi:10.1103/RevModPhys.54.729.

[27] J. D. Brown, "Action functionals for relativistic perfect fluids," Class. Quant. Grav. 10 (1993) 1579 doi:10.1088/0264-9381/10/8/017 arXiv:gr-qc/9304026].

[28] J. Klusoň, M. Oksanen and A. Tureanu, "Hamiltonian analysis of curvaturesquared gravity with or without conformal invariance," Phys. Rev. D 89 (2014) 064043 doi:10.1103/PhysRevD.89.064043 [arXiv:1311.4141 [hep-th]].

[29] P. Hořava, "Quantum gravity at a Lifshitz point," Phys. Rev. D 79 (2009) 084008 doi:10.1103/PhysRevD.79.084008 arXiv:0901.3775 [hep-th]]. 